

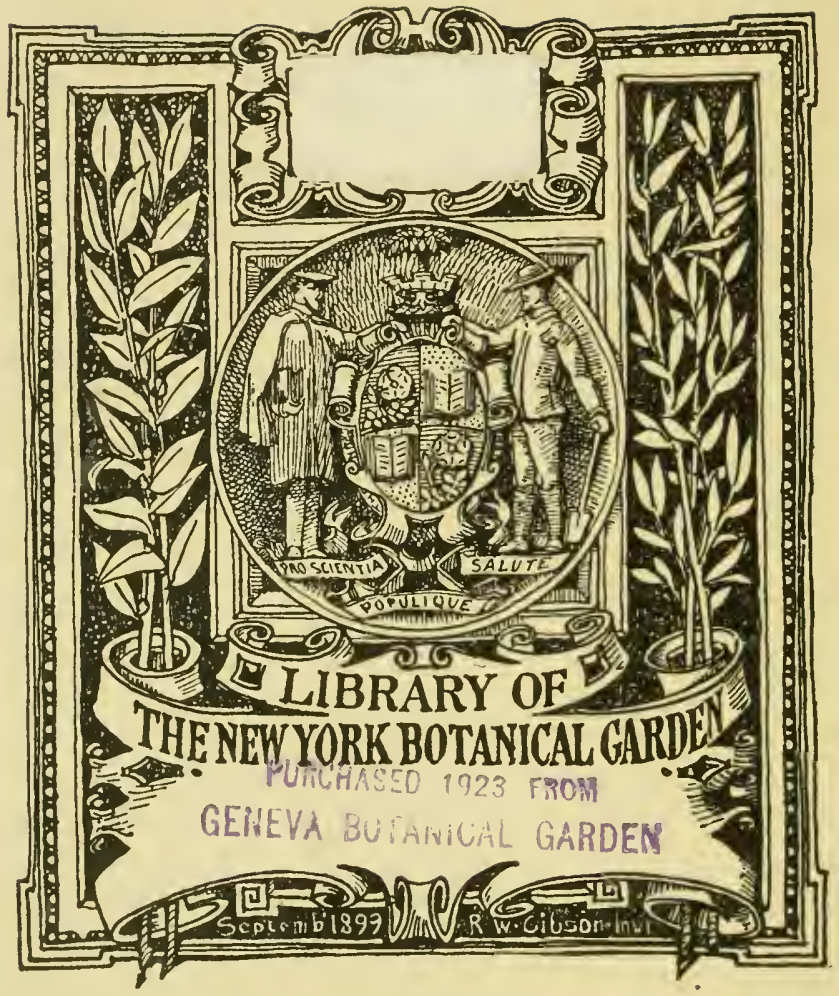




\section{Flora von Winterthur.}

Von

Dr. Robert Keller.

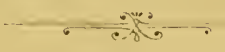

\section{Teil (I. Hälfte).}

Die Standorte der in der Umgebung von Winterthur wildwachsenden Phanerogamen, sowie der Adventivflora.

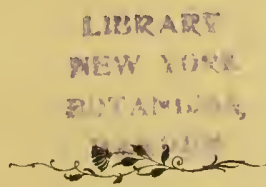

WINTERTHUR.

Buchdruckerei Geschwister Ziegler. 1891. 


$$
\begin{aligned}
& 0 \times 315 \\
& 1, x+3
\end{aligned}
$$

$$
\text { Helatel }
$$




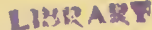 \\ NEW YORE \\ EOTAMCAC \\ GALUthe}

Die Pflanzenwelt meiner engern Heimat möehte ieh in der „Flora von Winterthur" von zwei Gesiehtspunkten aus darstellen, welehe naturgemïs zu einer Zweiteilung meiner Arbeit führen. Der erste, vorliegende Teil will eine mögliehst vollständige Uebersicht iiber die Standorte der in der Umgebung von Winterthur wildwachsenden Phanerogamen, sowie der Adventivflora bringen.

Die Gegend, auf welche sich das Standortsverzeichnis bezieht, umfasst nur die nähere $(5-8 \mathrm{Km}$.) Ungebung von Winterthur. Die Grenzpunkte unseres Gebietes sind also ungeführ die Ortschaften Hünikon in Nordwesten, Ober-Embrach im Westen, Winterberg im Südwesten, Weisslingen im Südosten and Rickenbach im Nordosten.

In das Verzeichnis habe ieh nur solehe Pflanzen aufgenommen, die ich selbst im Gebiete lebend sah, oder von denen mir Exsiceaten mitgeteilt wurden oder über deren Vorkommen ieh zurerlässige Angaben aus nenerer Zeit erhielt.

Die Hülfsquellen, die mir zu Gebote standen, sind folgende:

\section{A. Literatur.}

Gremli, Neue Beiträge zur Flora der Sehweiz, Heft 1-5. Die hier vorkommenden Angaben finden sich im Original in einzelnen der sub $B$ aufgeführten Hülfsmittel.

Herter, Die Flora in der Heimatkunde von Winterthur und Umgebung. 1887.

In den anmutigen Vegetationsbildern, in denen der Verfisser uns die heimische Flora vor Angen führt, werden zahlreiche Blütenpflanzen namhaft gemacht. Specielle Stand- 
ortsangaben finden sich jedoch nur in beschränkter Zahl. Zudem ist ein Teil derselben für das Standortsverzeichnis nicht verwertbar, da Herter in seiner eitirten Arbeit das Gebiet erheblich weiter fasst, als wie es oben umsehrieben wurde.

Jäggi, Eglisan in botanischer Bezichung. 1883.

Die östlichsten Standortsangaben greifen elsen noch in unser Gebiet ein.- Sie werden deshalb berücksicht.

Jäggi, Berichtüber neu e und wichtigere Beobachtungen, abgestattet von der Commission für die Flora von Deutschland. XXII. Schweiz in Berichte der deutschen botanischen Gesellschaft. Bd. VI und VII. $1888-90$.

Die in diesen Berichten erwïhnten Standorte unserer Umgebung finden sich ebenfalls in cinzelnen dor sub B erwiihnten Quellen.

Kölliker, Verzeichnis der phanerogamischen Gewächse des Kantons Zürich. 1839.

In dieser ersten Flora unseres Kantons werden etwas zu 300 specielle Standorte unseres Gebietes angeführt. Ich gebe aus dem Werke nur jene Arten in Kleindruck wieder, welche von Neuern nicht mehr beobachtet, zum Teil wol übersehen wurden.

Keller, Dr., Rob., Wilde Rosen des Kantons Zürich. Botanisches Centralblatt, Bd. XXXV. 1888.

Keller, Dr., Rob., Das Potentillarium von Herrn H. Siegfried in Winterthur. Botan. Centralblatt, 1889.

Keller, Dr., Rob., Beiträge zur schweizerischen Phanerogamenflora. II. Die Coniferemmistel. Botanisches Centralblatt, 1890.

\section{B. Handschriftliche Quellen.}

Cafisch, Notizen zur Phanerogamenflora von Winterthur. Standortsangaben von cirea 60 seltenercn Speeies. 
Herter, Ergiinzende Notizen zu oben eit. Publication.

Hug (†), Verzeichnis wildwachsender und kultivierter Arten aus der Umgebung von Winterthur.

Dasselbe umfasst 799 Nummern. Es entspricht zum grossen Teil dem Siegfried'schen Verzeichnis.

Siegfried, Ein einlissliches Verzeichnis, in welchem namentlieh verschiedene polymorphe Genera wie Potentilla, Epilobium, Cirsium, Mentha eingehende Berïcksichtigoung finden.

Steiner, Dr., Zur Flora von Winterthur, ein Verzeichnis von circa 300 meist weniger hïufiger Arten mit genauen Standortsangaben.

Weinmann, Dr. (†), Ein Standortsverzeichnis sehweizerischer, vorwiegend zürcherischer Phanerogamen, in welchem anch die hiesige Gegend einige Berïcksichtigung gefunden hat.

\section{Herbarien.}

Herbarium Hug. Die Mutter des zu früh verstorbenen, eifrigen Naturfreundes hat das 25 Bände umfassende Herbarinm in hochherziger Weise dem Mnseum Winterthur gesehenkt. Disselbe enthält die Belege zu den im Standortsverzeichnis aufgezählten Arten.

Herbarium Pfau. Eine kleinere Sammlung, die mir von meinem Freunde Richard Pfau g'eschenkt wurde. Die Zahl der aus dem Gebiete stammenden Exsiccaten ist jedoch keine grosse.

Herbarium Schellenbaum. Vor einigen Jahren habe ich dasselbe kïuflich erworben. Es enthält die Belege zu den Standortsangaben von Hirzel ( $\dagger$ ), Imhoof ( $\dagger$ ) und Schellenba $\operatorname{mon}(\dagger)$.

Herbarium Siegfried. Es enthält alle Belege zu den Standortsangaben Siegfrieds und ist namentlich reich an vielen interessanten Formen. 
Herbarium Ziegler. Dasselbe, 5 Bände umfassend, wurde mir kürzlich ron Herrn Redaktor Ziegler in liebenswürdiger Weise geschenkt. Es enthält die Belege einer Reihe seltenerer Arten, welche sein Soln Gottlieb $(\dot{T})$ in der Umgebung von Winterthur sammelte.

Sch-F. bedeutet Funde von Schülern. Ieh habe nur die durchaus zuverlässigen Standortsangaben aufgenommen.

Anmerkung. Das Herbarium von Lehrer Geilinger $(\dagger)$, welches vou Frln. Geilinger seiner Zeit dem hiesigen Lehrerinnenseminar geschenkt wurde, liess sich für die vorliegende Arbeit nicht verwerten, da die Standortsangaben fehlen.

Die Exsiccatennummern beziehen sich auf die in meinem Herbarium befindlichen Arten und Formen der Flora von Winterthur. Dasselbe enthält unter anderem ror allem zum ersten Male umfangreichere Belege zürcherischer Rosen und Rubi, speciell unseres Gebietes.

Wo niehts anderes bemerkt ist, entspricht die systematisehe Anordnung der von Nymann in scinem Cospectus Florae Europaeae befolgten.

Der zweite, später zu erscheinende Teil soll der Entwieklungsgesehichte unserer Flora gewidmet sein.

Allen denen, die mich durch ihre Mitteilungen bei der vorliegenden Arbeit förderten und in so bereitwilliger Weise unterstützten, spreche ich an dieser Stelle meinen aufrichtigen Dank aus.

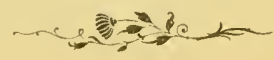




\section{Die Standorte}

der

in der Umgebung von Winterthur wildwachsenden Plinnerogamen, sowie der Adventivflora.

\section{Cl. I. Dicotyledoneæ.}

\section{Subcl. I. Thalamifloræ.}

I. Ranunculaceæ Juss.

Clematis Linné.

1. C. Vitalba L.

Exsicc. Nr. 1.

Gebüsche; überall.

Pulsatilla Mill.

2. P. vulgaris Mill.

Exsiec. Nr. 2, 2a, 2 b.

Sonnige Hügel. Nicht mehr so häufig wie früher.

Wolfensberg (Siegfried, Herter, Keller, Hug); Hoh-Wülflingen (Steiner, Ziegler, Herter, Siegfried, Keller, Hug); ob Töss (Siegfried); Neftenbach gegen den Radhof (Heer in herb. helv., Siegfried, Hug); Multberg (Keller); Waldwiesen ob Dynhard (Keller); Irehel ob Dättlikon (Herter); Hügel zwischen Seuzach und Hettlingen (Herter).

Var. fl. roseo.

Am Irehel (Steiner); Hoh-Wuilflingen (Herter, Siegfried, Hug).

\section{Anemone L.}

3. A. nemorosa $\mathrm{L}$.

Exsice. Nr. 3, 3a, 3 b.

Wälder, Gebüseh; uiberall, 


\section{f. rubriflora.}

Exsice. Nr. 4, 4 a.

Nieht selten unter der normalen Form.

Mit tiefroten Blüten in einem Wäldehen zwischen Seuzach und Lindberg (Keller); Wolfensberg (Siegfried); Hegiwald (Keller); Kyburger Schlossberg (Keller); hinter dem Hof Esehenberg gegen Kyburg (Herter).

Anmerkung: Bildungsabweichungen der Blüte: Bl. halbgefüllt (18-20 Pg.blätter); äussere Blütenhüllblätter laubblattartig. Exsice. Nr. 5.

Wolfensberg (Siegfried); Thurmhalde (Siegfried).

Bliite auf gleicher Höhe mit den Hïllblättern stehend; diese zu zwei.

Exsice. Nr. 6.

Kyburg (Keller).

4. A. ranunculoides L.

Exsicc. $\mathrm{Nr}$. 7, $7 \mathrm{a}, 7 \mathrm{~b}$.

An der Töss gegen die Kemptbrüeke (Hirzel); in der Au bei Rickenbaelı (Steiner, Herter); Bühl-IVinterthur (Imhoof, Keller), doeh wohl nicht spontan!

Thalictrum L.

5. Th. aquilegifolium L.

Exsice. $\mathrm{Nr}_{1}$. 8 .

Waldräinder, stellenweise häufig, doch nur zerstreut.

Waldungen an der Töss (Steiner); Linsetal: vom Sennhof bis hinunter zum Reitplatz häufig (Keller); Beerenherg (Herter); Eschenberg (Herter): Schlosstal (Herter); hinter dem Bruderhans (Hug); Brühlberg westlich rom Sehiessstand (Herter).

Ficaria Huds.

6. F. verna Huds.

Exsiee. Nr. 9, 9 a.

Wiesen, Heeken etc.; überall.

\section{Ranunculus L.}

7. R. aconitifolius L.

Exsice. Nr. 10, 10 a.

Ufer, sehr spärlich. 
Linsetal (Steiner, Herter, Sieg'fried); an der Töss unterhalb Kyburg (Caflisch); hinter dem Bruderhaus an der Töss (Hug); Wuhrland an der Töss unterhalb des Reitplatzes (Ziegler); zwischen der Eisenbahnbrücke iiber die Töss und dem Reitplatz (Keller); an der Kempt herwärts Kempttal (Keller); an der Zürcherstrasse nahe bei der Kemptbrïicke (Keller); an der Töss gegenüber der Schollenberger Mühle (Keller); beim Schlosshof (Caflisch).

8. R. nemorosus Dec.

Exsice. Nr. 11, 11 a.

W:älder.

Strassenbord zwischen Seen und Yberg (Schellenbanm); Wolfbühl über dem Schlosshof (Siegfried, Hug); Nord- und Südabhang von Hoh-Wülflingen nnd 'Tösserberg' (Siegfried); ob Neftembach (Siegfried); ob Dättlikon (Siegfried); Beerenberg (Siegfried); Schlossmïhle (Siegfried).

9. R. repens $\mathrm{L}$.

Exsice. $\mathrm{Nl} .12$.

An Gräben, in Aeckern, an Wegränden; überall.

Eine $f$. erecta mit aufrechten Stengeln hin und wieder an Gräben.

Exsice. Nr. 13.

Schützenwiese (Siegfried); an Gräben ansserhall, der Reitschnle (Keller).

10. R. montanus W.

Selten, an der Töss.

Linsetal an der Töss, von der frülıern Holzbrïeke flussabwärts am rechten Ufer (Siegfried, Herter).

11. R. acer L.

Exsice. Nr. 14.

Wiesen, Wege; überall.

12. R. auricomus $\mathrm{L}$.

Exsice. Nr. 1001.

Blumengäiten; selten.

In Walde bei Dynhard (Steiner); Hoffnumgsgnt-Winterthur (Ziegler). 
13. R. bulbosus L.

Exsicc. Nr. 15, 15 a.

Wiesen, Wegränder; iiberall.

14. R. Lingua L.

Exsice. Nr. 16.

Sumpfgräben; nicht häufig.

Ruchried (Siegfried); Gräben bei Rykon ob Kempttal (Sch-F.); Bodenlosenried bei Ober-Ohringen (Keller); Moosburg bei Effretikon (Caflisch).

15. R. Flammula L.

Exsicc. Nr. 17, 17 a.

Gräben, Sümpfe; nicht selten.

Wolfensberg (Siegfried, Keller); Ruchried (Keller); Riedwiesen um Neuburg (Keller); hinter der Gasfabrik (Siegfried); Riedwiesen bei Wiesendangen (Keller); zwischen Ohringen und Hettlingen (Hug).

16. R. arvensis $\mathrm{L}$.

Exsice. Nr. 18.

Aecker; häufig.

Batrachium S. F. Gray.

17. B. trichophyllum F. Sz.

Exsice. Nr. 19.

Stehende Gewässer, Gräben.

Mattenbach (Keller); Fenerweiher Ober-Ohringen (Hug, Siegfried); Feuerweiher Oberwinterthur (Keller); Ohringer Ried (Ziegler); Fenerweiher Reutlingen (Hug).

18. B. divaricatum Schur.

Exsice. Nr. 20.

Stehende Gewässer; selten.

Feuerweiher Ohringen (Siegfried).

19. B. heterophyllum Gray.

Exsicc. Nr. 21.

Stehende Gewässer; sehr selten.

Einmal im Kyburger Teich im Dorf (Hirzel). 
Nigella L.

20. N. arvensis $\mathrm{L}$.

Exsice. Nr. 22.

Aceker oder verschleppt; selten.

Auf Brachäckern gegen Henggart (Hirzel); Bodmersmühle (Herter).

Helleborus L.

21. H. viridis $\mathrm{L}$.

Exsicc. $\mathrm{N}_{1} .23$.

Gebüseh; selten.

Im Kyburger Schlossgraben hinter dem Gasthof (Siegfried, Pfau, Hug, Keller); im Gebüsch am Schloss Kyburg (Steiner, Caflisch); beim Exerzierplatz (Caflisch).

\section{Trollius L.}

22. T. europacus L.

Exsice. Nr. 24, 24 a.

Nasse Wiesen der Berge.

Kollbrunn (Siegfried); um Nussberg (Steiner); Yberg (Siegfried); Walkeweiher (Ziegler); zwischen Räterschen und Ricketwil (Keller); Breitensteinwiesen bei Dickbuch (Keller); Hettlinger Ried (Benz).

\section{Caltha L.}

23. C. palustris L.

Exsice. Nr. 25.

An Bächen; ïberall.

\section{Aquilegia L.}

24. A. vulgaris L.

Waldränder; selten.

Beim Walkeweiher sehr vereinzelt (Keller); zwischen Sonnenbühl und Brütten (Volkart).

f. atrata Koch.

Exsicc. Nr. 26, 26 a.

Waldränder, Gebüsche; häufig.

f. albifora. Selten.

Wiesen beim Wiesendanger Ried (Sch-F.). 
Aconitum L.

25. A. 7ycoctonum L.

Exsice. Nr. 27.

Tösserberg (Siegfried); Brühllberg (Weinmann); Abhang bei der Ruine Alt-Wülflingen (Steiner, Ziegler, Herter, Keller, Siegfried, Hug); im Walde bei Rickenbach (Steiner); Niederfeld-Pfungen (Caflisch); Beerenberg (Herter, Siegfried).

26. A. Napellus L.

Linsetal bei der Kyburger Brïcke (Siegfried). Herabgeschwemmt wie $R$. montanus.

\section{Delphinium L.}

27. D. Ajacis L.

Exsice. Nr. 28.

Bei der Sandgrube Neftenbach, Gartenflüchtling (Keller); zwischen Kollbrunn und Weisslingen (Hug).

28. D. Consolida L.

Exsicc. Nr. 29, 29 a.

Accker, hin und wieder.

Aecker vor Neftenbach (Keller); Pfungen (Hirzel); Henggart (Hirzel).

\section{Actæa L.}

29. A. spicata L.

Exsice. Nr. 30, 30 a, 30 b.

W:ilder; hin und wieder.

Brühlwald (Steiner, Siegfried); Eschenberg ob der Breite (Siegfried, Herter); Gamser (Ziegler); ob dem Gut (Keller); Ebnet (Caflisch); Schlosshof und hinauf gegen Alt-Wülflingen (Hug, Herter, Siegfried); Hoh-Wülflingen (Siegfried, Keller); Beerenberg (Siegfried, Caflisch); Kyburg (Keller); Tobel bei Rykon (Keller); Winterberger Steig (Keller).

\section{Berberider Vent.}

Berberis L.

30. B. vulgaris L.

Exsicc. Nr. 31, 31 a.

Gebüsche; häufig. 
Epimedium L.

31. E. alpinum L.

Exsice. Nr. 32, 32 a.

Bisweilen in Gïrten und von da aus verwildert.

Bühl hei Winterthur (Imhoof, Keller, Siegfried); Kyburger Schlosshalde (Sch-F., Siegfried).

\section{Nymphreacere Dec.}

\section{Nymphæa L.}

32. N. alba L.

Exsice. Nr. 33.

Stehende Gewïsser; micht selten.

Neuburger und Weihertaler Ried (Keller, Hug, Siegfried); Ruehried (Keller, Siegfried); Kyburg (Ziegler); Ruchried (Siegfried).

\section{Papaveracea Dec.}

\section{Papaver L.}

33. P. Rhoeas L.

Exsice. Nr. 34, 34 a.

Aecker, ungebaute Orte; häufig.

34. P. dubium L.

Exsicc. Nr. 35, 35 a.

Aecker; sclten.

In einem Getreideacker im Bühl bei Winterthur (Imhoof); in einem Schlage beim Egrgwald ob Wiesendangen (Keller).

35. P. Argemone L.

Exsiec. Nr. 36.

Aecker; selten.

Auf Aeckern bei Wülflingen (Hirzel).

\section{Chelidonium L.}

36. Ch. majus L.

Exsice. Nr. 37.

Auf Sehutt, in Hecken; tiberall. 


\section{Fumariacea Dec.}

Corydalis Dec.

37. C. cava Schw.

Exsice. Nr. 38, 38 a, 38 b.

Gebüsehe; herdenweise, doch nur an wenigen Standorten.

Bühl bei Winterthur (Schellenbaum, Imhoof, Keller, Siegfried); Sclanzengarten (Caflisch); am Kyburger Sehlossberg (Steiner, Keller, Hug, Cafliseh); im Gebüsch ob der Station Pfungen (Caflisch); Halde bei der Villa Ernst in Pfungen (Caflisch).

38. C. solida Sw.

Exsice. $\mathrm{Nr}$. 39.

Gebüsch; selten und wol nur angepflanzt.

Bühl bei Winterthur (Imhoof).

\section{Fumaria L.}

39. F. officinalis L.

Exsice. Nr. 40.

Auf Aeckern, Sehutt etc.; iiberall.

40. F. Vaillantii Lois.

Exsice. Nr. 41, 41 a.

Aecker; selten.

Sandplätze beim Bruni bei der Brücke von Pfungen (Hirzel). Auch Imhoof erwiihnt die Pflanze für Winterthur olme besoudere Standortsangabe.

\section{Crucifera Juss.}

Raphanistrum E.

41. R. imnocuum Med.

Exsice. $\mathrm{Nr}, 42$.

Aecker; überall.

Barbarea Br.

42. B. vulgaris $\mathrm{Br}$.

Exsice. Nr. 43.

Schuttstellen, Wegränder ete.; häufig: 


\section{Arabis L.}

43. A. hirsuta Scop.

Exsice. Nr. 44.

Hin und wieder an Wegen und Abhängen.

Schellenbaum ohne genauere Standortsangabe. An Abhängen und Wegen ïber der Ziegelei Neftenbach (Siegfried, Hug); Beeremberg in der Stöcklirüti (Siegfried); Kiesgrnben von Neftenbach (Siegfried, Hug).

Nasturtium $\mathrm{Br}$.

44. $\mathrm{N}$. officinale R. Br.

Exsice. Nr. 45, 45 a.

Bäche, Gräben; iiberall.

45. $N$. silvestre $\mathrm{R}$. Br.

Exsice. $\mathrm{Nr} .46$.

An Gräben; nicht selten.

An der Schützenstrasse gegen den Frohsim (Keller); zwisehen der Neuwiesemmïhle und Festhütte (Siegfried, Hug); im äussern Lindt bis zum Rosenberg (Siegfried, Keller); Ziegelei Rosenberg (Siegfried); beim Frolısinn gegen Veltheim (Siegfried); zwischen Wülflingen und Funkenbühl (Siegfried, Hug); an der Eulach hinter dem Technikum (Keller); Bahndamm beim Schlosshof (Siegfried).

46. $N$. palustre Dec.

Exsice. Nr. 47.

Feuchte Stellen.

An Grïben unterhalb des Schiitzenhauses (Imhoof); Ruchried, an der Strasse (Siegfried); Grïben in Güterbahnhof (Herter).

\section{Cardamine L.}

47. C. pratensis L.

Exsice. Nr. 48, 48 a.

Wiesen; iiberall.

48. C. amara L.

Exsice. Nr. 49, 49 a.

An Bächen.

Ufer der Eulach (Imhoof); in Ried zwischen Oberwinterthur und Mörsburg (Imhoof); Hesseng̈ütli (Siegfried); 
Nüihlebach bei der Festhütte (Herter, Siegfried); am Bach beim Fenerweiher im Hard (Siegfried); Gräben vor dem Eschenberger Hof (Koller).

49. C. hirsuta L.

Exsice. Nr. 50, 50 a, $50 \mathrm{~b}$.

An Wegriinderu, Acckern, namentlich in Weinbergen etc.; nicht selten.

Oberwinterthnrer Ried (Inhoof); Eschenberg (Imhoof); Wegränder um Winterthur (Ziegler); beim Lloydgarten (Keller); Gräben unterhalb der Weinberge vou Wülflingen (Keller); Lindberg (Hug); Wolfensberg (Siegfried); Haltenberg (Siegfried); Neuwiese (Siegfried).

50. C. silvatica Link.

Exsice. Nr. 51, 51 a, 51 b.

An Waldgräben; ziemlich selten.

Fenchte Gräben im Eschenberg (Imhoof, Ziegler); Waldsehläge ob der Breite (Siegfried, Hug); beim Haldengut (Siegfried, Keller).

51. Dentaria L.

D. digitata Lam.

Sehr selten.

Waldtobel unter Brütten (Steiner).

Hesperis L.

52. H. matronalis $\mathrm{L}$.

Exsice. Nr. 52.

Selten and wohl nur verwildert.

Am Eisenbahndamm oberhalb der Station Pfungen (Keller); an der Enlach (Schellenbaum, Siegfried).

\section{Alliaria Scop.}

53. A. officinalis Andrz.

Exsice. Nr. 53.

An Schuttstellen, Wegrïndern etc.; nicht selten.

Bodmersmuilhle (Siegfried); massenhaft an der Eulach vor der Giesserei und weiter abwärts (Keller, Herter, Siegfried); linter dem Künstlergiitli (Keller); Hard-Wülflingen (Hug). 


\section{Erysimum L.}

54. E. cheiranthoides L.

Exsice. Nr. 54-54 c.

Aecker; um Winterthur verbreitet.

Ob dem Bïhl-Winterthur (Imhoof, Ziegler); Aecker am Mattenbach (Herter); Grïze bei Winterthur (Steiner); Bodmersmühle (Siegfried); im Vogelsang (Siegfried); Schleife (Siegfried); an der Strasse nach Seen ausserhalb vom Mattenbach (Keller); in Seen (Keller); ansserhalb der Ziegelei Rosenberg (Keller).

Conringia Heist.

5). C. orientalis Rehb.

Exsice Nr. 55.

Selten und unbeständig in Aeckern, an Sehuttstellen.

Kehräcker bei Winterthur (Keller).

\section{Sisymbrium L.}

56. S. officinale Scop.

Exsice. Nr. 56.

Wegränder, Schuttstellen etc.; überall.

57. S. Thalianum Gay.

Exsice. Nr. 57, 57 a.

Nicht selten in Aeckern.

An der Strasse zum Bruderhaus (Imhoof); Aecker um Senzach (Keller); beim Rychenberg-Winterthur (Keller); zwischen Hard und Ziegelei Neftenbach (Hug); MaienriedWülflingen (Siegfried).

\section{Brassica L.}

58. B. oleracea $\mathrm{L}$.

Exsice. Nr. 58.

Kultiviert und verwildert; an Schuttstellen, Ufern ete. nicht selten.

59. B. Napus L.

Exsice. Nr. 59.

Kultiviert und verwildert: wie vor. 
60. B. Rapa L.

Exsiec. Nr. 60.

Kultiviert und verwildert; wie vor.

Sinapis L.

61. S. arvensis L.

Exsice. Nr. 61.

Aecker; häufig.

62. S. alba L.

Exsice. $\mathrm{Nr}$. 62.

Aecker; selten.

Beim Wollenhof.Winterthur (Schellenbaum).

Erucastrum Br.

63. E. Pollichii Schimper.

Exsice. Nr. 63-63 d.

Schuttstellen, Ufer' nicht häufig.

An der Töss beim Hard (Imhoof, Hug); an der Töss (Herter); bei der Mühle Neuwiese (Siegfried); an der Töss zwischen Dorf Töss und Schlosshof (Siegfried, Hug); Schuttstellen hinter dem Primarschulhaus (Sch-F.); Eisenbahndamm bei der Kemptmündung (Keller); an der Eulach (Keller); an der Töss beim Reitplatz (Keller); Lind (Sch-F.); ausserhalb Hettlingen (Hug); Eulach-Schützenwiese (Hug, Siegfried).

Diplotaxis De.

64. D. muralis Dc.

Selten; auf Schutt.

Bodnersmühle (Herter).

Berteroa Dc.

65. B. incana Dec.

Exsice. $\mathrm{Nr} \cdot 64$.

Schuttstellen; selten.

Bodmersmühle (Siegfried, Herter); Bahndamm beim Schlossh of (Siegfried).

\section{Cochlearia L.}

66. C. Armoriaca L.

Exsice. $\mathrm{Nr}_{\mathrm{r}}, 65$.

Ufer, Wegränder; nicht lıäufig. 
An der Eulaeh bei der Obermülle (Schellenbaum); Tössufer bei der Spinnerei Rieter (Siegfried); im ZelgliWinterthur (Keller).

\section{Erophila De.}

67. E. verna E. Mey.

Aecker, Grasplätze etc.; sehr häufig und formenreich.

\section{f. majuscula Jordan.}

Exsiec. Nr. 66.

Wiilflingen (Imhoof); Pfungen unterhalb der Station (Keller); Reitplatz (Keller); Ziegelei Neftenbach (Hug); Wülflingen (Hng); Hard (Siegfried); Maienried (Siegfried).

f. stenocarpa Jordan.

Exsice. Nr. 67.

Reitplatz (Keller); Aceker ansserhalb der Düngerfabrik Winterthur' (Keller).

f. precox Stev.

Fxsiec. Nr. 68.

Eisenbahndamm zwisehen Pfungen u. Wülflingen (Keller) Rosenberg (Hug, Siegfried).

\section{Alyssum L.}

68. A. calycinum L.

Exsice. Nr. 69-69 e.

Somnige Stellen; nicht selten.

Zwischen Wülflingen und Pfungen (Hirzel); Kiesgrube in der Grüze (Hirzel, Keller, Imhoof); Schlosshof (Keller, Herter, Hug); Eisenbahndamm gegeniiber vom Reitplatz (Keller); Bodmersmuihle (Herter); Neftenbach (Siegfried).

\section{Camelina $\mathrm{Cr}$.}

69. C. sativa $\mathrm{Cr}$.

Exsice. Nr. 70.

Aecker; selten.

Hinter der Hardfabrik (Hirzel); Veltheim (Siegfried); in Flachsickern Wülflingen (Herter). 


\section{Iberis $\mathrm{L}$.}

70. I. umbellata L.

Exsice. Nr. 71.

Hin und wieder als Gartenflüchtling zu beobachten. An der Töss (Hirzel).

71. I. pinnata L.

Exsice. Nr. 1004.

Selten.

Aecker beim ehemaligen Lärchenwäldchen-Winterthur (Ziegler).

72. I. amara L.

Exsice. Nr. 72-72 c.

In Aeckeru; nicht häufig.

Schlosshof gegen Pfungen (Hirzel); Ruchegg (Imhoof); Mörsburg reichlich (Keller); Ohringen (Keller); zwischen Kyburg und First (Keller); um Hegi (Steiner); Neftenbach (Siegfried); Töss (Siegfried); Breitehof (Siegfried).

\section{Thlaspi L.}

73. Th. arvense $\mathrm{L}$.

Exsice. Nr. 73, 73 a.

Aecker; überall.

74. Th. perfoliatum L.

Exsicc. Nr. 74.

Wegränder ete.; iiberall.

\section{Lepidium L.}

75. L. Draba L.

Exsice. Nr. 1003.

Schuttstellen; selten.

Bodmersmühle (Siegfried, Horter); neue Strasse beim Eschenberghof (Ziegler).

76. L. sativum L.

Exsicc. Nr. 75.

Angebaut und hin und wieder verwildert.

77. L. campestre R. Br.

Exsice. Nr. 76.

In Aeckern, an Wegrändern u. s. f.; hin und wieder. 
Linsetal (Hirzel); an der Seemerstrasse (Hirzel) ; im Rosenberg. (Siegfried); in der Kiesgrube von Veltheim (Siegfried); Neftenbach (Siegfried).

Capsella Mönch.

78. C. bursa pastoris Mch.

Exsice. Ni. 77.

Wegränder, Accker; iiberall.

f. integrifolia Heg. Die ganzblätterige Modifieation der Art. Exsice. Ni. 78.

Findet sich an gleichen Stellen wie der Typus, jedoch zienlich selten.

Schellenbaum ohne specielle Angabe des Fundortes.

\section{Isatis $\mathrm{L}$.}

79. I. tinctoria L.

Exsicc. Nr. 1002.

Dämme, selten; versehleppt.

Am Kanal zwischen Sennhof und Kyburg (Ziegler, Caflisch).

\section{Resedacen Dc.}

\section{Reseda L.}

80. R. lutea L.

Exsice. N1. 79.

WTegränder, Ufer.

Wülflingen (Weinmann); an kiesigen Stellen der Töss bei Pfungen (Imhoof); an der Töss (Jäggi); beim Hardberg' (Herter); reichlich $11 \mathrm{~m}$ die Zicgelei Neftenbach (Siegfried, Keller); zwisehen Hard und Ziegelei Neftenbach (Busch, Siegfried); Weinberge Oschwang bei Neftenbach (Keller'); im Dorfe Seen (Siegfried); Rosenberg-Veltheim (Siegfried); ansserhalb Hettlingen (Hug; Siegfried).

81. R. Tuteola L.

Wie vorige; selten.

Wïlflingen (Weinmann). 


\section{Cistinea De.}

Helianthemum G.

82. H. vulgare $\mathrm{G}$.

An trockenen, sonnigen Stellen; überall.

\section{Violariex De.}

\section{Viola $\mathrm{L}$.}

83. V. mirabilis L.

Exsicc. Nr. $81-81 \mathrm{~d}$.

In den Bergwäldern ziemlich häufig.

Irchel ob Dättlikon (Schellenbaum); Hoh-Wülflingen gegen Neuburg (Imhoof, Herter, Hug); Suidseite des Tösserberges zwischen Dättnau und Neuburg (Siegfried, Keller); am Taggenberg unterhalb Wülflingen (Herter, Siegfried, Hug); am Beerenberg ob dem Hard (Keller); im Linsetal (Keller); im Gebüsch an der Tüss gegen Kyburg (Steiner); Kyburg (Herter).

$V$. mirabilis L. $\times V$. silvatica $\mathrm{Fr}$.

Südabhang des Tössberges gegen Dättnan (Siegfrier).

84. V. silvatica Fr.

Exsice. Nr. 82, 82 a, 83 b.

Wälder, Gebüsche; häufig.

forma 7 . albo.

Exsice. Nr. 83.

Selten.

Am Bruderhauserweg (Imhoof).

85. V. Riviniana Rehb.

Exsice. Nr. 84.

Wie vorige, aber viel seltener.

Im Wäldehen ob der Breite (Siegfried); am Wolfensberg gerade im Anfang in den feuchten Beständen links und rechts von der Strasse (Siegfried, Hng); im Walde ob der Station Seuzach (Keller); Eschenberg ob der Schinderhü̈tte (Hug). 
86. $V$. odorata L.

Exsice. $\mathrm{Nr}$. 85.

Banmgärten, Hecken, Bördern ete.; häufig.

87. V. alba Bess.

Selten.

Ohringen (Siegfried); Hettlingen (Herter); Wolfensberg (Herter); über Velthein links ron der Strasse zum Wolfensberg).

88. V. hirta L.

Exsice. Nr. 86.

An Bördern, Grasplätzen ete.; überall.

$V$. hirta L. $>V$. ordorata L.

Links an der Strasse iiber Veltheim an den grasigen Abhängen innerhalb des ersten Rebgelaindes (Siegfried).

89. V. collina Besser.

Exsice. Nr. 87, 87 a, 87 b.

Wiilder; ziemlich selten.

Am Fusse des Wülflinger Schlossberges (Imhoof); HohWiilflingen (Imhoof, Herter, Siegfried, Hug); Beerenberg (Keller); Wolfensberg (Hug, Siegfried): Abhïnge von Brütten gegen das Diittnau (Keller).

90. V. tricolor L.

Exsice. Nr. 88.

Felder; häufig.

\section{Droseraceit De.}

Drosera L.

91. D. Tongifolia Huds.

Exsice. Nr. 8?.

In Sumpfwiesen; selten.

Im Ruchried vor Hettlingen rechts von der Strasse Unterohringen-Neftenbach vor dem Bahnübergang und links nach dem Bahnübergang (Keller, Siegfried); Wiesendanger Ried (Steiner); im Ried bei Hettlingen (Hug, Caflisch, Herter).

92. D. rotundifolia L.

Sumpfwiesen; selten.

Wieșendanger Ried ('Steiner). 
Parnassia L.

93. I. palustris L.

Exsice. Nr. 90.

Nisse VViesen, Gräben; häutig.

\section{Polygalea Juss.}

\section{Polygala L.}

94. P. Chamcebuxus L.

Exsice. Nr. 91-91 d.

W:alder; nicht selten.

Sonnige Stellen beim Hegiwald (Keller); auf dem Brïhlberg (Imhoof, Keller, Herter); in 'Tobel gegen die hintere Hub ob Neftenbach (Keller); ob der Rotfarb Neftenbach (Keller, Siegfried); in Brühlbachtobel (Keller); am Kyburger Schlossberg (Keller, Herter); bei Huggenberg im October blühend (Keller); Hoh-Wülflingen (Hug); Beerenberg (Siegfried, Herter); Multberg (Herter).

f. rhodoptera Bennet.

Zwischen Wartgut und Daittlikon (Siegfried).

95. P. comosa Schrk.

Exsice. Nr. 92, 92 a.

Hügel; nicht selten an steinigen, sonnigen Stellen.

Hol-Wülflingen (A. u. R. Keller, Siegfried, Herter, Hug); Wolfensberg (Siegfried, Hug, Herter, Keller); Becrenlerg' (Siegfried, Herter, Hug); Tössberg (Hug').

96. P. vulgaris Selırk.

Exsicc. Nr. 93.

Seltener als vorige.

Feuchte Waldstellen (Schellenbaum); Hoh-TVülflingen (Siegfried); Wolfensberg (Siegfried, Hug); Beerenberg (Siegfried).

97. P. austriaca $\mathrm{Cr}$.

Exsicc. Nr. 94, 94 a.

Ueberall in Triften, an Bördern etc.; rot- und weissblïhend bedeutend seltener als blaublithend.

f. fl. albo.

Wolfensberg ob Veltheim häufig (Herter). 
XII. Silenacese Lindl.

Githago Dsf.

98. G. segetum Dsf.

Exsice. Nr. 95.

Unter Getreide; überall.

\section{Lychnis L.}

99. L. floscuculi L.

Exsice. Nr. 96, 96 a, $96 \mathrm{~b}$.

Feuchte Wiesen; überall.

Melandrium Rœhl.

100. M. silvestre Rohl.

Exsice. Nr. 97.

Neuwiese-Winterthur an der Wartstrasse (Siegfried); Eulach: rechtes Ufer am jähen Abhang bei der Schützenwiese (Siegfried).

101. M. pratense Røhl.

Exsice. Nr. 98.

An der Eulach-Hessenguitli (Hug); Eulach linkes Ufer bei der Schuitzenwiese nüher der Stadt (Siegfried); Rosenberg am Strässchen gegen den Walkeweiher (Siegfried).

Silene L.

102. S. inflata Sm.

Exsice. Nr. 99, 99 a.

Wiesen; gemein.

103. S. nutans L.

Exsice. Nr. 100.

Waldränder, Hügel ; häutig.

104. st. noctiflora L.

Exsice. Nr. 101.

Aecker; nicht häufig.

Getreideäcker im Bühl bei Winterthur (Imhoof, Herter', Siegfried, Hug); Ober-Winterthur (Steiner, IFirzel, Keller); Ohringen (Hirzel); Vogelsang bei Winterthur (Ziegler).

105. s. gallica L.

Eschenberg, im Krebsbachtobel (Caflisch). 


\section{Saponaria L.}

106. S. Vaccaria L.

Exsice. Nr. 102.

Unter Getreide; nicht häufig.

Beim Feuerweiher in Ohringen (Siegfried); an einem Weg im Eschenberg (Imhoof, Weinmann); Finsteri im Eschenberg bei Winterthur (Ziegler); Aecker gegen Seen läufig (Herter); Neftenbach (Steincr); linkes Tössufer gegen den Sehlosshof (Hug); Töss (Siegfried).

107. S. officinalis L.

Exsiec. Nr, 103.

Ufer; stellenweise hänfig.

Wülflingen (Weinmann); an der Eulach (Siegfried); Schützenwiese, an der Eulach (Herter, Hug, Sch-F.); an der Töss (Steiner, Herter, Siegfried); bei der Eisenbahnbrücke der Zürreherlinie über die Töss am linken Ufer reichlich (Keller).

Gypsophila L.

108. G. muralis L.

Exsice. Nr. 104.

Gräben, Mauern, Aceker; nicht häufig.

In einem Strassengraben hinter dem Rosenberg (Hirzel).

\section{Dianthas L.}

109. D. Armeria L.

Exsice. Nr. 105.

Waldränder; selten.

Beim Seemer-Pol an Walde (Hirzel); auf dem Irchel (Hirzel); ob den Häusern der Breite links im Wäldehen im lichten Bestande (Siegfried); Waldrand ob Pfungen (Steiner); Waldrand ob Wiesendangen (Keller).

110. D. Carthusianorum L.

Exsice. Nr. 106.

Hügel; nicht häufig.

Brühlberg (Steiner, Weinmann, Herter, Ziegler); Wolfensberg (Herter); am Waldrande zwischen der Schollenbergermühle und Sehlosshof am linken Tössufer (Siegfried); am Waldrande bei Ottikon (Keller); längs der Töss (Herter). 
111. D. superbus L.

Hügel; selten.

Kemleten (Caffliseh).

D. prolifer L.

Dättlikon (Jïggi).

XIII. Alsinacen Bartl.

Malachium Fr'.

112. M. aquaticum $\mathrm{Fr}$.

Exsice. Nr. 107, 107 a.

Grïiben; gemein.

Cerastium L.

113. C. arvense L.

Exsice. Nr. 108.

Wegräinder, Wiesen; selten.

Bei Henggart (Schellenbaum); Ziegelei Neftenbach in einer Wiese und am Fussweg der Kiesgrube Auental zur Ziegelei Neftenbach reichlich (Siegfried); Aceker unter der Glockengiesserei Neftenbach (Herter); Somnenberg bei Winterthur (Ziegler).

114. C. triviale Lk.

Exsice. NI. 109.

Mauern, Wegränder etc.; überall.

115. C. viscosum L.

Exsice. Nr. 110.

Wegränder, Aecker etc.; nicht häufig.

Im Vogelsang (Imhoof); am Bahndamm im Vogelsang (Siegfried); ïber der Breite in den abgeholzten Stellen (Siegfried); in der Griuze (Keller).

116. C. glutinosum Fr.

Selten.

Ohringen in der Nähe von Bettwiesenrierl (Sięfried).

117. C. semidecandrum I.

Exsiec. Nr. 111.

Nicht häufig.

Eschenberg (Imhoof). 
Stellaria L.

118. St. nemorum L.

Selten.

Waldrand ob der Breite (Siegfried); Walkeweiher (Siegfried).

119. St. media Cyr.

Exsice. Nr. 112.

Wegränder, Aecker; gemein.

120. St. holostea L.

Exsice. Nr. 113.

Hecken; selten.

Gegeniiber der Locomotivfabrik an der Strasse nach Töss (Gisler stud. med., Huğ, Keller, Siegfried, Herter).

121. St. graminea L.

Exsice. Nr. 114.

Hecken etc.; häufig.

122. St. uliginosa Murr.

Exsicc. Nr. 115.

Auf kultivierten Stellen im Eschenberg (Schellenbaum). Mohringia L.

123. M. trinervia Clairv.

Exsice. Nr. 116.

Nicht überall.

Feuchte Waldstellen im Eschenberg bei Winterthur (Imhoof); an Hecken über der Turmlialde (Siegfried); am Waldrand ob der Hub (Siegfried); bei der Ruine AltWülflingen (Hug, Siegfried).

M. muscosa L.

Steiner in Köll. Phan.

Arenaria L.

124. A. serpyllifolia.

Exsice. Nr. 117.

An Mauern, Aeckern; überall.

Alsine L.

125. A. tenuifolia L.

Exsice. Nr. 118.

Nicht häufig. 
Auf Aeckern bei Ober-Ohringen (Hirzel); an Eisenbahndamm beim Sehlosshof' (Siegfried); Neftenbach (Jäggi). Sagina L.

126. S. procumbens L.

Exsiec. Nr. 1006.

Längs der Manern vom Wartgut (Siegfried): Manern beim Wartbad (Hug); Schlosshof bei Kylurg (Ziegler).

Spergula L.

127. Sp. arvensis L.

Exsice. Nr. 119.

Aecker; nicht häufig.

Auf dem Grüzenfeld gegen Rümikon (Hirzel); Bahndamm bei der Kemptbrïeke (Siegfried): Sclinderluïtte (Siegfried); auf dem Stadtschutt (Siegfried).

\section{Lines De.}

\section{Linum L.}

128. L. tenuifolium L.

Exsice. Nr. 120, 120 a.

Sonnige Hügel; selten.

Am Irehel bei Dättlikon (Hirzel, Steiner, Herter); zwischen Wartgut und Dïttlikon reehts vom Striissehen an Abhang reichlich (Siegfried, Ing); an der Brahalde bei Hünikon (Keller).

129. L. catharticum L.

Exsice. Nr. 121, 121 a.

Wiesen, steinige Plätze etc.; ïberall.

\section{Malvaces $\mathrm{Br}$.}

130. Hibiscus Trionum L.

Exsice. Nr. 122.

Seltene und nur vorübergehende, eingeschleppte Ruderalpflanze.

In einem Brachacker vom Hard gegen Pfungen (Hirzel); einmal im Garten (Herter). 
Althaea L.

131. A. hirsuta L.

Seltene und unbestiindige Ruderalpflanze.

Bei der Bodmersmuilhle (Herter).

\section{Malva.}

132. M. Alcea L.

Exsice. $\mathrm{Nr} \cdot 123$.

Selten.

Bei Humlikon (Pfau); Kyburg (Ziegler).

133. M. moschata L.

Exsice. Nr. 124, 124 a, 124b.

Wegränder ete.; ziemlich selten.

Auf der Griize an der Landstrasse gegen Rümikon (Hirzel); links von der Strasse zwischen Kemptbrücke und Bahnwärterhäuschen (Sicgfried); am Eisenbahndamm nahe bei der Kemptmiundung (Keller); zwischen Winterthur und Wülflingen (Keller); Neftenbach (Siegfried).

134. M. silvestris L.

Exsice. Nr. 125, 125 a.

Wegränder, Schutt; hin und wieder.

Unbebaute Plätze gegen das Ruchegg. (Hirzel); Rebgelände links vom Fussweg zwischen der Kiesgrube Auental zur Ziegelei Neftenbach (Siegfried); beim Stadtmist (Keller); Kyburg (Ziegler); an Bördern um Dättlikon (Steiner); Eulach-Schützenwiese (Hug, O. Liechti, Siegfried).

135. M. rotundifolia L.

Exsice. $\mathrm{Nr} .126$.

Schuttstellen, WVegriinder ete.; ziemlich lıänfig.

Lind beim Bahnübergang (Siegfried, Hug); in Sennhof in der Nähe von Gärten (angepflanzt?) häufig (Keller); Kyburg links von der Landstrasse bein Eingang in's Dorf (Keller).

Tilia L.

\section{Tiliacere Juss.}

136. T. platyphyllos Scop.

Exsice. $\mathrm{Nr}_{1} .127$.

W älder ; verbreitet. 
Briilllberg (Siegfried, Keller); Lindberg (Siegfried, Keller); Hoh-Wülflingen (Siegfried, Keller).

137. T. parvifolia Ehrl.

Exsice. Nr. 128.

Wälder; wie vorige.

\section{Hypericinea De.}

\section{Hypericum L.}

138. H. montanum L.

Exsice. Nr. 129.

Eschenberg (Siegfried); Lindberg (Siegfried); Tössberg. (Siegfried); Brühlberg (Siegfried, Keller); auf dem hintern Wolfensberg (Keller); Hol-Wülflingen (Steiner); Weg vom Schlosshof nach der Ruine (Herter).

139. II. hirsutum L.

Exsice. Nr. 130, 130 a.

Nicht selten in unsern Wäldern.

Beim Schlosshof (Schellenbaum, Herter); um Kyburg (Pfau, Keller); Rosenbergregen den Walk ew eiher (Siegfried); im Eschenber'g über der Breite massenhaft (Siegfried, Herter); Brühlberg an den Abhängen hinter den alten Eichen (Siegfried): an der Eulach, Brücke bei der Festhı̈̈tte (Siegfried); Bülll (Herter); im Bähntal bei Kollbrunn vor der Teufelskirehe reichlich (Keller); Wolfensberg (Hug).

140. H. tetrapterum $\mathrm{Fr}$.

Exsiec. Nr. 131-131 c.

Gräben; gemein.

141. II. quadrangulum L.

Exsiec. Nr. 132.

Waldschläge; nicht häufig.

Eschenberg (Siegfried); Lindloerg (Siegfried); Seen (Siegfried); Brühlberg (Siegfried).

H. quadrangulum $\mathrm{K} . \times$ H. tetrapterum $\mathrm{Fr}$.

Exsicc. Nr. 133.

Eschenberg: Waldschläge ob (ler Breite (Siegfried). 
142. H. perforatum L.

Exsice. Nr. 134, 134 a.

An Gräben, lichten Waldstellen; gemein.

var. veronense Schrk.

Hinter den alten Eichen im Tobel anf dem Brühlberg (Siegfried). Schmalblätterige Formen, die den Uebergang zum Typus herstellen, hin und wieder z. B. im Vogelsang bei Winterthur (Keller).

H. perforatum L. $\times$ H. quadrangulum L.

Eschenberg: ob der Breite (Siegfried).

143. H. humifusum $\mathrm{L}$.

Exsice. Nr. 135.

Lichte Waldstellen; selten.

Auf dem Hegiberg gegen Rümikon (Hirzel); Elsau (Bucher).

\section{Acerinen De.}

Acer L.

144. A. Pseudo-Platanus L.

Exsice. Nr. 136, 136 a, 136 b.

Nicht selten in Wiildern, doeh zumeist angepflanzt.

Lindberg ob dem Haldengut (Keller); unterhalb HohWülflingen (Keller).

var. vitifolium Tausch.

Exsice. Nr. 137.

Lindberg: am Waldrande ob dem Haldengut gegen das Gütli (Keller').

145. A. platanoides L.

Exsice. Nr. 138, 138 a.

Wälder, verbreitet, jedoch zumeist angebant.

146. A. campestre L.

Exsice. Nr. 139, 139 a, 139 b.

Geboiische, Laubhölzer; nicht selten.

Am Tössrain (Hirzel, Keller); Schlosshof (Hirzel, Keller); Beerenberg, Hardberg an der Töss (Herter); am linken Tössufer (Siegfried). 


\section{Geraniacen De.}

Geranium L.

147. G. sanguineum L.

Exsice. Nr. 140.

Hügel; verhreitet.

Tössberg Südseite längs des Grates gegen Hoh-Wülflingen (Siegfried, Herter, Hug, Keller); Wolfensberg (Siegfried, Herter, Keller); Brühlberg Südabhang gegen die Töss (Siegfried, Herter); Bälmtal bei Kollbrunn (Keller); Irehel (Steiner, Siegfried); Lindberg (Hng); Beerenberg (Herter, Siegfried); obere Hub (Siegfried).

148. G. palustre L.

Exsiec. Nr. 141.

Nicht häufig.

An der Eulach (Siegfried, Keller); links von der Strasse bei der Snlzer'schen H'abrik gegen die Breite (Siegfried); Ottikon-Kyburge (Hng); Vogelsang (Rich. Ernst).

149. G. pheerm L.

Exsice. $\mathrm{Nr}$. 142.

Selten und vielleicht nur verwildert.

Beim Bülll in der Langgasse Wintertlur (Steiner, Ziegler, Siegfried, Hug, Keller, Herter).

150. G. pyrenaicum L.

Exsice. Nr. 143.

Selten.

Beim Haldengut an der Strasse (Siegfried, Keller, Herter); Wiesen an der Schaffhanserstrasse (Herter).

151. G. columbinum L.

Exsicc. Nr. 144.

Häufig an Sehuttstellen, Feldern ete.

152. G. dissectum L.

Exsice. Nr. 145.

Wie vorige.

153. G. pusillum L.

Exsice. Nr. 146.

Häufig auf Schutt. 
154. G. Robertianum L.

Exsice. Nr. 147.

Schattige Stellen; iberall.

Erodium l'Her.

15). E. cicutarium l'Her.

Exsice. Nr. 148.

Auf den Wegen im Adlergarten-Winterthur (Hirzel)

Wolfensberg: in Aeckern (Keller), Südabhang hie und da (Siegfried); Fussweg vom Auental zur Ziegelei Neftenbach (Siegfried).

XX. Balsaminea. A. Roch.

\section{Impatiens L.}

156. I. noli tangere L.

Exsice. Nr. 149.

Feuchte, schattige Orte; an wenigen Stellen, da aber heerdenweise.

Eschenberg (Siegfried, Herter); am Graben in der Turmhalde (Steiner, Keller, Siegfried); an Bruderhausweg (Keller); oberhalb vom Waldegg in Unmasse (Keller); beim Kugelfang-Winterthur (Keller, Siegfried); bei der Mündung des Weisslinger Baches in die Töss (Keller); Brühlber"ğ (Steiner, Siegfried).

157. I. parviflora Dc.

Exsice. Nr. 150.

Selten.

Im Eschenberg (Siegfried); Gartenunkraut links an der Paulstrasse Winterthur (Keller); Roscnberg in einer Schuttgrube (Hug, Siegfried). .

\section{Oxalidere De.}

\section{Oxalis L.}

158. O. Acetosella L.

Exsice. Nr. 151.

Feuchte, schattige Stellen; überall. 
159. O. stricta L.

Exsice. Nr. 152, 152 a.

Auf bebauten Stellen; hin und wieder.

Am Eisenbahndamm in Vogelsang (Siegfried); Somnenberg-Winterthur (Imhoof); in der Pflanzschule-Winterthur (A. Keller); im alten Kirchhof-Winterthur St. Georgen (Hug, Keller, Herter); an der Eulach bei der Brüicke auf der Sehützenwiese (Hug, Siegfried).

\section{Subclassis II. Calycifloræ.}

XXII. Celastrinea Br.

\section{Staphylea L.}

160. St. pinnate L.

Exsice. Nr. 153.

Selten und wahrscheinlich nur verwildert.

Todtental am Fuss von Hoh-Wülflingen (Ziegler, Herter); beim Sehlosshof (Siegfried); Alt-Wülflingen (Siegfried, Ifug, Keller, Herter); etwas unterhalb Hoh-Wülflingen (Steiner, Keller); Pfungen (Herter).

\section{Evonymus L.}

161. E. vulgaris Scop.

Exsice. Nr. 154.

Gebüsche; gemein.

Ilex L.

162. I. aquifolium L.

Exsice. Nr. 155.

W:ilder; verbreitet.

Auf dem Tössberg (Keller); im Lindberg am Weg gegen Rentlingen (Keller); im Brühllbachtobel bei Semnhof (Keller); im Tobel von Rykon (Keller); Wolfensberg(Herter); Nordalbhimg von Hoh-Wülffingen (Herter). 


\section{Rhammere Br.}

Rhamnus L.

163. Rh. Frangula L.

Exsice. N1. 156.

Wïlder, Gebüsche; gemein.

164 R. cathartica L.

Exsice. Nr. 157.

An steinigen, buschigen Waldstellen nicht selten.

Hessengiitli (Hirzel); Tössberg(Keller, Siegfried); Brïlılbachtobel bei Sennhof (Keller); Eschenberg (Steiner, Siegfried).

\section{Papilionacea L.}

\section{Genista L.}

165). G. germanica L.

Exsice. Nr. 158, $158 \mathrm{a}, 158 \mathrm{~b}$.

Hügel; verbreitet.

Tössberg (Siegfried, Keller); Hoh-Wülflingen (Steiner, Imhoof, Siegfried, P. Liechti, Keller, Herter); Wolfensberg' Herter, Siegfried, Hug); Beerenberg (Siegfried); Ir'chel bei der Hub (Siegfried); Winterbergersteig (Keller); Brülılbachtoluel (Keller).

166. G. pilosa L.

Sonnige Stellen; selten.

Irchel (Steiner').

167. G. tinctoria L.

Exsicc. Nr. 159.

Nicht häufig:

Vom Schlosshof bis Pfungen auf der Suidseite (Hirzel); Irehel (Steiner); auf der Hub und zwischen Wartgut und Dättlikon (Siegfried, Hug, Herter); im Bähntal bei Kollbrunn rechts von der Strasse nach Nussberg (Keller).

168. G. sagittalis L.

Exsice. Nr. 160.

Auf dem Grat nach Hoh-Wülflingen (Siegfried, Herter, Hng, Keller); an einem Wäldchen zwischen Märsburg nnd 
den Riedwiesen ausserhalb Ruchegg (Steiner, Keller, Herter); iu Brühlbachtobel ob Sennhof (Keller); Irehel (Herter, Siegfried).

\section{Ononis L.}

169. 0 . arvensis L.

Exsice. $\mathrm{Nr}$. 161.

Wegränder etc.; ïberall.

170. O. campestris K. Z.

Exsice. Nr. 162.

An trockenen Stellen; selten.

Wolfensberg (Siegfried).

\section{Anthyllis L.}

171. A. Vulneraria L.

Exsiec. Nr. 163.

Trockene Wiesen; iiberall.

\section{Medicago L.}

172. M. falcata L.

Exsice. Nr. 164, 164 a.

Wege, Raine.

Linkes Tössufer zwischen Töss und Schlosshof (Siegfried, Hugi); an der Töss unterhalb vom Hard (Imhoof); Strassenbord bein Ruchegg (Keller).

173. M. sativa L.

Exsicc. Nr. 165.

Sehr häufig an WVegrändern, Wiesen; verwildert.

174. $M$. media P.

Exsicc. Nr. 166.

Mit vorigen; nicht sehr häutig:

Am linken 'Tössufer zwischen Töss und Sehlosshof' reichlich (Siegfried); Wegräinder und Strassenborde beim Ruchegg (Keller).

175. M. minima Desr.

Pfungen an einem Fussweg bei der Station gegen das Dorf Pfungen (Siegfried); bei der Tuchfabrik (Caflisch). 
176. M. Lupulina L.

Exsice. Nr. 167.

Wegräinder etc.; ïbrrall.

Melilotus Juss.

177. M. officinalis Dess.

Exsice. Nr. 168.

Wegränder etc.; häufig.

178. M. altissima $\mathrm{Tl}$.

Exsicc. Nr. 169.

Weniger verbreitet als vorige.

Massenhaft in der Lehmgrube ob Veltheim beim Rosenberg' (Siegfried, Hug); Brïlllberg (Sch-F.).

179. M. alba Desi.

Exsicc. Nr. 170.

Wegränder etc.; sehr häufig.

Trifolium L.

180. T. rubens L.

Exsicc. Nr. 171.

Sonnige Hügel; selten.

Am Tössberg auf dem Grat gegen Hoh-Wülflingen (Herter, Steiner, Siegfried, Hug, Keller); am Beerenberg (Siegfried); im Täli über Dättlikon am Irchel (Steiner, Siegfried, Herter).

181. T. alpestre $\mathrm{L}$.

Am Irchel (Steiner).

182. T. medium L.

Exsicc. Nr. 172, 172 a.

Wälder; nicht selten.

Tössberg (Siegfried, Keller); Holı-Wülflingen (Siegfried, Keller, Herter); Wolfensberg (Siegfried, Hug, Keller, Herter'); Beerenberg (Siegfried); Brülılbachtobel ob Sennhof (Keller); in Bähntal bei Kollbrum (Keller); im Eggwald bei Wiesendangen (Keller); Bolsternbuck bei Kollbrum (Keller); am Steigbach bei Weisslingen (Keller): Eschenberg (Siegfried). 
183. 'T. pratense L.

Exsice. Nr. 173.

Wiesen, Wegränder etc.; gemein.

184. T. incarnatum L.

Exsice. Nr. 174.

Selten und nur verwildert.

Vereinzelt in einer Waldwiese am Eschenberg (Schellenbaum); Aecker um Aesch (Steiner).

185. T. arvense $\mathrm{L}$.

Exsice. $\mathrm{Nr} .175$.

Selten.

Wolfensberg (Herter, Siegfried).

186. T. fragiferum L.

Exsice. $\mathrm{Nr} .176$.

Selten.

Am Fussweg von Dättnau nach Brïtten (Hirzel); unterhalb Pfungen an der Töss (Hirzel); an Kammerwegen in den Weinbergen unterhalb vom Wartbad (Keller).

187. T. montanum L.

Exsice. Nr. 177.

In Waldwiesen, an Waldrändern; sehr häufig.

188. 'T. hybridum L.

Exsice. Nr. 178.

Nicht lıäufig.

Linkes Tössufer gegenüber dem roten Hüsli (Siegfried, Herter); Lindberg an Fussweg nach Reutlingen (Keller).

189. T. repens $\mathrm{L}$.

Exsicc. N1. 179.

Grasplätze, Wege; gemein.

190. T. procumbers L.

Exsice. $\mathrm{Nr}$. 180, 180 a.

Aecker, WVegränder ete.; häufig.

191. T. minus Sm.

Exsice. $\mathrm{Nr} .181,181$ a.

Auf trockenen Wiesen; nicht sehr häufig. 
Am Bord der Eulach gegen das Sehützenhaus (Hirzel); im Bühl bei Winterthur (Imhoof); bei der Kemptbrücke (Siegfricd); im Kies linter der Gasfabrik (Siegfried).

\section{Lotus L.}

192. L. siliquosus L.

Feuchte Stellen; selten.

Am Irchel (Steiner).

193. L. uliginosus Schk.

Éxsiec. Nr. 182.

Am sumpfigen Waldstellen nicht selten.

Massenhaft über der Breite am Bache (Siegfried); beim Walkeweiher (Siegfried); am Strassengraben beim Ohringer Ried (Keller); am Wiesendanger Bach (Keller); Gräben zwischen Ohringen und Winterthur (Hug, Siegfried); Eschenberg (Siegfried).

194. L. corniculatus L.

Exsice. Nr. 183.

Tegränder, trockene Wiesen; gemein.

f. pilosa.

Exsiec. Nr. 184, 184 a.

\section{Selten.}

Die Haare an den Blättern reichlich, am Stengel spärlich und mehr anliegend, sind kürzer als an der behaarten Form des insubrischen Florengebietes.

Brühlberg (Imhoof); bei Dättlikon (Imhıof).

\section{Coronilla.}

195. C. varia L.

Exsice. Nl. 185.

Nicht selten.

Nagelschmiede bei der Kemptbrücke (Siegfried); Station Kempttal (Keller); bei der Eisenbahnbrüicke über die Töss (Keller); Reitplatz (Siegfried, Keller); Wolfensberg (Siegfried, Keller, Herter); Station Wülflingen (Siegfried, Keller): Lindberg beim Alpgütli (Seh-F., Keller); am Waldrande des Breitenloo bei Wiesendangen sehr reichlich (Keller); Eggwald bcim Ruchegg (Keller); Oberwinterthur (Steiner); 
Bodmersmiilhle (Herter); von der Station Wiesendangen nach Mörsburg (Herter).

\section{Hippocrepis L.}

196. II. comosa $\mathrm{L}$.

Exsice. Nr. 186.

Somnige Orte; sehr häufig.

Colutea L.

197. C. arborescens $\mathrm{L}$.

Gebuisch; spontan?

Am Rande des Brülıwaldes (Steincr).

Astragalus L.

198. A. glycophyllus L.

Exsice. Nr. 187.

Wälder; ziemlich verbreitet.

Breite (Siegfried); Wolfensberg (Siegfried); Hoh-Wülflingen (Siegfried); Kemptbrücke (Siegfried); Tössrain-Linsetal (Keller, Herter); Winterberger Steig (Keller); Kyburg (Ziegler); zwisehen Kollbrunn und Weisslingen (Hug); Ebnet (Caflisch); Eschenberg (Caflisch, Siegfried).

\section{Onobrychis L.}

199. O. sativa Lam.

Exsice. Nr. 188.

Trockene Hügel etc.; häufig.

\section{Lathyrus L.}

200. L. latifolius L.

Exsice. Nr. 189, 189 a.

Selten und nur verwildert.

Hinter dem Hessengütli gegen Wuilflingen (Hirzel); am Lindberg beim Steinbruch über Oberwinterthur (Keller).

201. L. silvestris L.

Exsice. Nr. 190-190 d.

Wälder; verbreitet.

Beim Hessengütli im Wäldehen an der Eulach (Hirzel); am Warthügel (Hirzel); Eschenberg (Imhoof); ob der Breite (Siegfried); an den Abhängen bei der Kemptbrïcke 
(Siegfried); Kyburg (Steiner, Siegfried); Alt-Wülflingen (Keller, Siegfried, Herter) ; Winterberger Steig vor Kempttal (Keller); Strassenbord ausserhalb des Ruchegg (Keller); am Brühł (Steiner, Hug, Siegfried); Waldrand ob Wiesendangen (Herter).

202. L. tuberosus.

Exsice. Nr. 191.

Nicht häufig.

Aecker bei Dättlikon (Schellenbaum).

L. palustris $\mathrm{L}$.

In Köll. Phanerog.

203. L. pratensis L.

Exsice. Nr. 192.

Wiesen; überall.

204. L. Nissolia L.

Exsice. Nr. 193, 193 a.

In Aeckern; sehr selten.

Beim Dorf Breite bei der Kapelle (Hirzel); Aecker ob dem Haldengut an Waldrande des Lindberges (Keller).

205. L. Aphaca L.

Exsice. Nr. 194, 194 a, 194 b.

Aecker; nicht selten.

Ohringen (Hirzel); Reutlingen (Hirzel); Neftenbach (Hirzel, Keller); Pfungen bein Bruni (Steiner, Schellenbaum); in der Eigelhart (Keller); Hochwacht-Winterthur (Hug); im Hündler-Töss (Caflisch).

Orobus L.

206. O. vernus $\mathrm{L}$.

Exsice. Nr. 195.

Wälder; sehr häufig.

207. O. niger L.

Exsice. Nr. 196, 196 a.

Stellenweise häufig.

Irchel (Steiner); Reitplatz links an der Strasse (Siegfried); auf dem Wolfensberg reichlich (Siegfried, Hug; 
Keller); Brühllbachtobel bei Semnhof (Keller); Beerenberg (Cafliseh); Ebnet (Cafliseh); am Fussweg vom Rossberg nach Kämleten reiehlich (Cafliseh).

208. O. tuberosus L.

Exsiec. Nr. 197.

Wälder; lä̈utig.

Vicia L.

209. V. dumetorum L.

Exsiec. Nr. 198.

Linsetal (Steiner); Eschenberg (Inhoof); iiber der Breite an der Waldstrasse häufig (Siegfricd); zwisethen Kemptbrüeke und Kempttal häufig (Siegtried); am Hügel der Schlossruine Alt-Wülflingen (Herter, Hug); zwisehen Sennhof und Kyburg (Hug).

210. V. silvatica L.

Exsice. Nr. 199.

Im Walde ob Pfungen (Steiner); Abliänge von AltWülflingen gegen das Tälchen zum Schlosshof (Siegfried).

211. V. tenuifolia Rth.

Da und dort unter Getreide (Siegfried).

212. V. Cracca L.

Exsiee. Nr. 200.

In Heeken, Wegränder ete.; gemein.

213. V. sepium L.

Exsice. Nr. 201.

In Wiesen ete.; gemein.

f. flore albo.

Exsice. Nr. 20:2.

Selten.

Am Weg vom Sehlosshof naeh Alt-Wülflingen mahe beim Bahnwärterhäusehen (Keller).

214. I. sativa L.

Exsice. Nr. 203.

Unter Getreide sehr häutig. 
215. V. angustifolia Reich.

Exsice. Nr. 204, 204 a.

Unter Getreide.

Ohringen (Siegfried); Aecker am Lindberg (Keller).

\section{Ervum L.}

216. E. hirsutum L.

Exsice. Nr. 205, 205 a.

Aeker; ziemlich hïufig.

Aecker um Seen (Hirzel); Eschenberg (Keller).

217. E. tetraspermum L.

Exsice. Nr. 206, 206 a.

Aecker; ziemlich häufig.

Felder um Winterthur (Schellenbaum); Aecker um Seen (Keller); Eschenberg ob der Breite (Hug).

\section{Drupacese L.}

\section{Prunus L.}

218. P. Padus L.

Exsiec. Nr. 207, $207 \mathrm{al}, 207 \mathrm{~b}$.

Gebüsch; hin und wieder.

Im Gebuisch an der Eulach (Steiner); Linsetal (Schellenbaum); Tössberg (Siegfried); am Kyburger Schlossberg (Ziegler); bei der Kyburger Brücke (Keller); ob Ricketwyl (Keller).

219. P. avium L.

Exsice. Nr. 208.

Waldränder, Hügel; überall.

220. P. Cerasus L.

Exsice. Nr. 209.

Nicht häutig.

Brühl (Imhoof); Süidseite des Wolfensberges (Siegfried); am Haltenberg ob dem Hard (Siegfried); Hoh-Wülflingen (Hug).

221. P. spinosa L.

Exsice. NT, 210.

Ueberall. 


\section{Ord. Senticosa L.}

Spiræa L.

222. Sp. Aruncus L.

Exsiec. Nr. 211, 211 a.

Bergwälder; nieht solten.

Brühlwald (Steiner); Eschenberg (Keller); Lindberg (Keller); Kyburger Schlosshalde (Keller); Hoh-Wülflingen (H11

2.23. sp. Ulmaria L.

Exsiee. Nr. 212.

An Gräben; uiberall.

224. Sp. Filipendula L.

Esiee. Nr. 213.

Feuehte Waldwiesen; selten.

Reitplatz an der Töss (Steiner); bei Ohringen gegen Veltheim (Hirzel); Wolfensberg (Herter); bei Kämleten (Keller).

\section{Rubus L. *)}

2.5. R. saxatilis L.

Exsice. Nr. 214-214e.

Bergwälder.

Im lintern Esehenberger Wald im Steinbachtobel; Brühlbaehtobel; Ablänge gegenuiber der Station Sennhof; Rykoner Tobel; Tössrain.

2:6. R. Idceus L.

Exsice. Nr. 215, 215a, 215 b.

Wälder'; nieht selten.

Lindberg ob dem Walkeweiher, beim Bäumli etc; Esehenberg vor dem Essehenbgerhof ete.; Brühllbachtobel ete.

क) Der Anordnung liegt Focke's s'ynopsis Ruborum Germanice zu Grunde. Sämmtliche Standortsangaben fussen anf den Beobachtungen des Verfassers. Die meisten der eitierten Arten und Formen hat Herr Prof. Favrat durchgesehen. Für seine Bemühungen sei ihm anch an dieser Stelle aufrichtig gedankt. 
227. R. sulcatus Vest.

Exsice. $\mathrm{Nr} .216,216$ a, 216 b.

Trälder; selten.

Lindlserg: ob dew Haldengut gegen den Walkeweiher; anf dem Holzschlag hinter dem Bäumli; ob dem Giitli am Fussweg nach Reutlingen vor dem Beginn des Hochwaldes; Holzschlag ob dem Tössertobel links rom Fahrweg nach dem Süsenber's.

․ㅡ. R. Mercieri G. Gener.

Exsice. $\mathrm{Nr}, 217$.

Wolfensberg ob Veltheim.

2.29. R. thyrsoideus Spec. collect. Exsice. $\mathrm{Nr}_{\mathrm{r}}$. 218-218c.

Dem R.thyrsoidens wird von den Schriftstellem ein verschiedener Umfang zugeschrieben. Focke erklärt ihn als Sammelart, "deren abweichendste Formen allerdings anch als getrennte Arten betrachtet werden können....., welche durch Mittelformen, die namentlich läng's der Alpen ... verbreitet sind, verbunden werden." Unter dem Collectivnamen nemne ich hier jene Formen, welche mir nicht so scharf differenciert zu sein scheinen, dass sie mit Sicherheit mit einem der drei gut ausgeprägten Typen - R. candicans Weihe, R. thyrsanthus Focke und $R$. elatior Focke - in welchen der $R$. thyrsoideus sens. coll. bei uns auftritt, zu identificieren wären.

Lindberg beim Bäumli; Brühlbachtobel ob Semnhof; Wolfensberg bei Veltheim; Eschenberg: Querstrasse rom Vogelsang zur Bruderhausstrasse.

f. virescens mihi.

Exsice. Nr. 219.

Lindberg: hinter dem Baumli am Weg gegen OberWinterthur.

Schössling tiefgefurcht, kahl; Stacheln miissig zahlreich, \&-10 im Interfolium. Blätter fünfzählig gefingert. Blattstiel spärlich behaart; Endblättchen breit eiförmig zugespitzt, an der Basis schwach herzförmig. Alle Blättchen dentlich gestielt; Stiel der untern Seitenblättchen $8 \mathrm{~mm}$; oberseits kahl, unterseits locker kurzhaarig, blassgriin, seidenglänzend. Blätter der Bliitenaxe unterseits z. T. hellgrün, locker behaart, z. T. (die oberen) grauweiss filzig. Fruchtknoten an der Spitze mit einem lockern Hanrschopf: - Eine rerkahlende Form des Typus. 
230. R. candicans Weihe.

Exsicc. Nr. 220, 220 al, 220 b.

Wälder; in typiseher Ausbildung selten.

Wolfensberg bei Veltheim; im Eschenberg in etwas breitblättriger Form; Winterberger Steig ror Kempttal.

2:31. R. thrysanthus Focke.

Exsice. Nr. 미 $1-221$ p.

Wälder; verbreitet.

Wolfensberg ob Veltheim; Ebnet ob Töss; Eschenberg: am Waldrand ob dem Vogelsang; auf dem Platean des Brühlberg und oberhalb vom Guitsch; Lindberg: am Weg nach Reutlingen, am Weg vom Bäumli nach Oberwinterthur; Winterberger Steig vor Kempttal; Broülllbachtobel ob Semnliof.

Anmerkung. Sebmalblätterige Formen, die an den meisten der erwähnten Standorte neben den typischen beobachtet werdem. bilden Uebergänge zu $R$. candicans Weihe.

29:2. R. elatior Focke.

Exsice. Nr. 2.202, 2020‥

Selten.

Beim Steinbruch ob den Weinbergen von Reuthingen mit fast zottig behaartem Sehössling: mit spärlicherer Behaarung der Axe im Eschenberg.

293. R. bifrons Vest.

Exsice. Nr. 223-223k.

Waldränder; ziemlich häıfig.

Im Lindberg am Weg nach Rentlingen beim Ausgang aus dem Hochwald; hinter dem Bäumli; Eschenberg: vor dem Vogelsang, vor dem Eschenberghof; ob Töss anf dem Ebnet; Wolfensbers: ob Veltheim, beim Steinbrueh.

2:) 1. R. macrostemon Focke.

Exsiec. Nr. 224, 224 a, 224 b.

Selten.

Kyburger Rain, rechts an der Landstrasse; Lindbers hinter dem Bänmli; im Bähntal bei Kollbrumn. 
235. R. obtusangulus Grml.

Exsicc. Nr. 225-225 e.

Nicht häufig.

Wolfensberg, etwas oberhalb der Veltheimer Steinbrïche; Lindberg an der Strasse vom Walkeweiher nordwärts gegen Seuzach, ebenso am Wege nach Reutlingen: zwischen Ruchegg und Mörshurg am Rande des äusserm Riedes; Winterberger Steig vor Kempttil.

236. R. tumidus Gremli.

Exsice. $\mathrm{Nr} .226$.

\section{Selten.}

Vom Brülılberg liegt mir ein Specimen vor, das nach den Sternhiärchen, die anf der Blattoberfläche zerstrent sind, und dem rimnigen Blattstiele zu urteilen ein Abkömmling des R. tomentosus Borlkh. ist. Mit R. obtusangulus Grml. und R.tumidus Grml. stimmt unsere Form in der Fruchtbarkeit iiberein, kann also nicht ein primärer Bastard des R. tomentosns Borkh. sein, demn diese sind "in der Regel sehr wenig fruchtbar."

Mit Gremlis obiger Species stimmt unser Specimen darin überein, dass das Endblättchen rundlich-herzförmig (grösste Länge $9_{98} \mathrm{~cm}$., grösste Breite $9_{88} \mathrm{~cm}$.) ist und eine etwa $8 \mathrm{~mm}$. lange anfgesetzte Spitze trägt. Dagegen sind die mnteren Seitenblïttchen kaum kürzer gestielt als bei $R$. obtusangulus Grml. Hier sind die Stielchen $0,2-0,4$ cm. lang, d. h. 6-15 mal kürzer als der Stiel des Mittelblättchens, und etwa 10-25 mal kürzer als das zugehörige Blättchen. Der Stiel der untern Seitenblättchen ist an vorliegender Form 5 mal kleiner als der des Mittelblättcliens und 12 mal kürzer als das zugehörige Blättchen.

237. R. tomentosus Borkhausen.

1. vulgaris Focke.

a) canescens Focke $=R$. tom, var. canescens Wirt.

Exsice. Nr. 227-227 d.

Wälder; zerstreut, fast selten.

Eschenberg an der Strasse zum Bruderlaus; Winterberger Steig: Formen, deren Schösslingsaxe nur vereinzelte Stieldriisen trägt, neben Formen, die den Uebergang zu R. setoso-glandulosus Wirt. bilden; ob dem VogelsangWinterthur an der Strasse durch's frühere Lärchenwäldchen. 
b) inter canescentem el glabratum.

Hxciec. $\mathrm{Nl}_{1} \cdot 228-228 \mathrm{r}$.

Die vielen Formen des $R$. tomentosus, die je dnrch muleiche Stiirke der Pubescenz und durch Verschiedenheit in der Zishl der Stieldriisen und Stachelchen von einander abweichen, stollen in muserem Florengebiete keine selbststindig gewordenen Typen dar. Sie sind viehmehr die durch zahlreiche Zwischenformen mit einander verbundenen Erscheinungsformen des gleichen Typus. Die sub $b$ zu nemnenden Formen sind oberseits grïn, jedoch nicht völlig kahl. Thre Axe ist kahl und drïsenlos oder sie besitzt doch nur in spärlichem Grade Haare mo Stieldriisen. Bestachehung gleich.

Die Form findet sich an glejchen Stellen mit a. u. с. Briilılbachtobel ol, Semnhof; Winterberger Steig vor Kempttal; hier auch in Formen mit ziemlich reichlicher Drïsigkeit und feiner Bestachelung der Axen; an der Querstrasse vom Vogelsang zur Bruderhausstrasse.

c) glabratus Focke $=\mathrm{R}$. tomentosus var. glabratus (iodr. Exsice. Nr. 229, 229 a, 209 b.

Mit $a$ und $b$, doele seltenes als heide.

Briilhlbachtobel ob Senuhof; Winterhererer Strig vor Kempttal; im Bähntal an der Strasse nach Nussheren.

2. setoso-glandulosus Wirtg.

a) canescens $=\mathrm{R}$. cinerens lichb.

Exsice. Nr. 230.

Winterberger Steig vor Kempttil.

b) ylabratus $=$ R. Lloydianus G. Crener.

Exsice. Nr. 231,231 a.

Biilntal, an der Strasse von Kollbrmm nach Nussluerge; Winterberger Steig ror Kempttal.

3. villicaulis Gremli.

a) canescens.

Die Combination der oberseits aschgraufilzigen Blatter mit dieht filzig-zottiger Axe habe ich bisher in Gebirte nicht heoh:ichtet.

b) glebratus.

Exsiec. Nr. 232, 232. a, 232 b.

Selten.

Winterberger Steig ror Kenupttal: Biiluntal, an der strisse vou Kollhrum nach Nussloreg. 


\section{Bastarde des R. tomentosus Borkhausen.}

\section{a) Stieldrüsenlose Formen.}

\section{R. tomentosus Borkh. $\times R$. bifrons Vest.}

Exsicc. Nr. 233-233f.

Auf dem Plateau des Brühlberges bei Winterthur; im Brühlbachtobel ob Semhof, hier in Formen, die ziemlich reichliche Fruchtentwickhung zeigen; Winterberger Steig vor Kempttal; an der Querstrasse vom Vogelsang-Winterthur zur Bruderhausstrasse.

R. tomentosus Borkh. $\times$ R. thyrsoidens sens. coll. an macrostemon?

Exsice. N1'. 234, 234 a.

Hierher ziehe ich zwei Formen, welche von den vorigen durch den stark gefurehten Schössling, central entspringende, stärker gezahnte Blättchen und gekrïmmte Stacheln an der Blütenstandsaxe verschieden sind.

Vogelsang bei Winterthur; Bolsterenbuck ob Kollbrumu.

b) Stieldrüsenführende Formen.

R. tomentosus Borkh. $\times R$. Radula Wh. Exsice. N1. 235.

Schössling kantig gefurcht, zerstreut behaart, Bchaarumg aus Sternhäärchen, Büschelhaaren und Striegelhararen bestehend; mit vereinzelten kurzen Stieldrüsen; Stacheln gleich, ziemlich kraiftig, gerade; Stachelchen fehlend. Blätter meist fussförmig, 5 zäblig; mere Seitenbläittchen kurz gestielt, oft fast am Grunde der Stiele der obern Seitenblättchen entspringend, Nebenblätter drüsig gewimpert; Blattstiel rinnig, behaart und stieldriisig; Mittelblättchen eiförmig oder rundlich eiförmig, an Grunde ansgerandet oder meist deutlich berzförmig, zugespitzt, oberseits fast kahl, unterseits filzig; Zahnung grob, ungleichmässig, Spreite vorn oft etwas eingeschnitten. Blütenaxe kantig, etwas gefurcht, abstehend behaart, abwärts fast zottig; Stachehn ziemlich lang, obere meist gerade, stark nach rückwärts gerichtet; Sticldrüsen an den obern Teilen des Blütenstandes ziemlich zahlreich, aber kurz. Blätter der Blütenstandsaxe 5- oder meist 3 zithlig, obere einfach und diese wenigstens mit zerstrenten Sternhäärchen. Endblättehen der 3 zählig'en Blätter bisweilen keilig. Rispeniiste ansgebreitet; Rispe bisweilen reichlich durebblïttert. Blütenstiele dicht behaart, mit kurzen in der Bebaarung fast verborgeneu Stieldrïsen und Iangen Stachelchen. Kelchblätter nach der $\Lambda$ n. there zurückgeselılagen. Blumenblitter ziemlich gross, breit- 
eiförmig, aussen ziemlich dicht behaart, inmen fast kahl, blassrosa. Staubgefässe den Griffeln gleich hoch oder dieselben wenig iiberragend. Fruchtknoten behaart.

Hub; Winterberger Steig vor Kempttal.

238. R. vestitus Weilie et Nees.

Exsice. Nr. 236-236 g.

Wälder’; verbreitet.

Briilılbachtobel ob Sennhof an somnigen, offenen Stellen; Winterberger Steig vor Kempttal; Eschenberg: am Waldrand ob dem Vogelsang, Strasse zum Eschenberghof; Lindberg: Tössertobel, ob Rentlingen, beim Walkeweiher.

239. R. conspicuus P. J. Müiller.

Exsice. $\mathrm{Nl} .237$.

Selten.

Lindberg hinter dem Bïumli.

240. R. semivestitus Favrat.

Exsice. $\mathrm{Nr}, 238$.

Selten.

Ein Speeimen, das ich im August 1887 auf dem Platean des Brühlberg sammelte, will Favrat in sched. obiger Art unterorduen.

241. R. teretiusculus Kalt.

Exsice. Nr. 239-239g.

Lindberg: am Wege nach Reutlingen, hinter dem Bäumli; Eschenberg: vor dem Eschenberghof an der Strasse zum Aussichtsturm, ob dem Vogelsanğ; anf denı Wolfensberg ob Veltheim; Winterbergerer Steig ror Kempttal.

f. umbrosa.

Exsice. Nr. 240, 240 a, 240 b.

Im Hochwald.

Von der typischen Form dureh die dümmen, last häutigen Blätter und die erheblich schwächere Pubescenz verschieden.

Lindberg, am Fussweg nach Rontlingen; in linteren Eschenberger Wald.

f. valde villosa Favrat in sched.

Exsice. N1: 241.

In Jahre 1888 hahe ich Herrn Farat, dem ansgezeichneten Kenner der westschweizerischen linbi, eine Form zur Einsicht 
geschickt, die mir eine Varietät des lis restitus W. et $N$. zu sein schien. Farrat hielt dieselbe für eine $f$. valde villosa des $R$. teretinseulus. Gremli glanlst, wie F, mir sclureibt, es sei eine gute Art. Die Frage muss leider vor der Hand eine offene bleiben, da der Standort, rechts an der Landstrasse von Sennhof nach lyburg, einige hundert Schritte ob der gedeckten Brücke, eincr liutschung wegen verschwunden ist und ich seither umsonst in der Nilie nach einer ähnlichen Form suchte. Nachfolgend dic Buschreibnug' (nach meinen Exsiccaten):

Schössling kantig, flachseitig, kräftig, dichtzottig behaart. Behaarung mit ziemlich zahlreichen Stieldrüsen nutermischt; Stacheln fast gleich; schwach, behaart; Blätter 3 zählig oder fussförmig 5z zählig. Blattsticl fast zottig behaart mit geraden oder schwach gebogenen riickwärts gerichtecen feinen Stacheln bewehrt, wenig kïrzer als clas Endblittchen. Nebenblätter etwas breit. Blättchen diek, an Rande ungleich gesägt und gewimpert; oberseits mit anliegender ziemlich dichter glänzender Behanrung"; unterseits sammtartig, weich, durch die dicht abstehende seidengliinzende Behaarung. Endblättchen rundlich, zugespitzt, an der Basis schwach herzförmig, bis 4 mal länger als sein Stielchen. Bliitenstandsaxe dicht behaart, zottig mit zahlreichen geraden teinen Stachelchen bewehrt, Stieldrüsen kürzer als die alsstehenden Haare. Blätter 3 zälılig, Seitenblättchen sehr kurz gestielt, fist sitzend, ron der Pubescenz der Schösslingsblätter; olere blïtentragende Aestchen fast rechtwinklig abstehend, reichlich bewehrt; Blütenstielchen filzig-zottig; lïngere Stieldriisen die al)stehenden Haare überragend; Kelch filzig-zottig, mit abstehenden Haaren, welche die reichlich vorkommenden Stieldrüsen iiberragen; Kelchzipfel an der Frucht zurïckgeschlagen; Kronenblätter eiförmig, gegen die Basis keilig, beiderseits kurzhaarig. Staubgefässe (Filamente wud Antheren) behatr, zahlieich, die Griffel iiberragend; Fruchtknoten kahl.

242. R. suavifolius: Gremli. Exsiec. Nr. 24:2, 242 a.

Lindberog bei Winterthur; Eschenberg: am WValdrand ob den Vogelsang-TVintertlıne.

24:3. R. Radula Weihe et Nees.

Exsice. Nr. 243-243 c.

Wolfensberg im Veltheimer Steinbrinch; Brïlılbachtobel ol Sennhof; an der Strasse von Nusslyergo nach Selilatt; im Eschenbersy besi Winterthme. 
244. R. liudtis Wrihe et Nees.

Exsice. Nr. $244-244 \mathrm{c}$.

Brühlberg bei Winterthur am IIorizontalweg hei den grossen Eichen; Eschenberg: an der letzten Querstrasse vor dem Hof' zum Eschenberg; Esehenbergstrasse; Lindberg: an Wege nach Reutlingen.

245. Li. saltuum Focke.

Exsice. Nr. $245-245 \mathrm{~d}$.

Brühlberg bei Winterthur; ob Reutlingr'n im Lindberog; ob Töss auf dem Ebnet; Wolfensberg bei Veltheim.

246. Li. Weiheanus Gremli.

Exsice. Nr. 246.

Brühlberg bei Winterthur. - Nahestehende Formen anch aus dem Eschenberg und Lindberg.

247. R. Guentheri W. et N.

Exsice. Nr. 247.

Vogelsang; im Brühlbachtobel ob semnhof.

248. R. Villarsianus Focke.

Exsice. Nr. 248-248 e.

Wälder; ziemlich häutig.

Winterberger Steig vor Kempttal; im Elmet ob 'Tüss: Esehenberg bei Winterthur; Lindberg: am Wegr zum Bäumli im Tössertobel, beim Walkeweiher.

249. Li. dumetorum sens. coll.

Exsice. Nr. 249-2 $49 \mathrm{~g}$.

Hïntig.

Hecken an der Strasse rom Haldengut zum Roscrnberg; Lindberg: hinter dem Bäunli anf der gerodeten Waldstelle, im Tössertohel; Eschenherg; in der schw:ïzi bei Hettlingen; Winterberger Steig vor Kempttal; im Brühlbachtobel oh Semuhof; am Kyburger Rain; ob den Wreinbergen von Rickenbach; an der Brahlalde bei Hünikon; (o) Kollbrumn gegen Wreisslingen.

250. li. ceesius L.

Exsice. Nr. 250.

Ueberill. 


\section{f. glandulosa.}

Exsice. Nr. 251, 251 a.

Nit der Normalform; verbreitet.

Bei der Hansermülle unterhalb Töss.

\section{f. armata.}

Exsice. Nr. 252.

Mit der Normalform; verbreitet.

Hausermühle unterhalb Töss.

R. cresius L. $\times R$. Idceus, f. superciesius.

Exsice. Nr. 253.

Thalhein gegen Dynhard.

$R$. cresius L. $\times R$. tomentosus Borkh.

Exsice. Nr. 254-2541.

Am Wege vom Gütli ob dem Rychenberg-Winterthur gegen die Haldenstrasse; hinter dem Bäumli links von der Strasse längs des Waldrandes; an der Brahalde bei Hünikon; am Walkeweiher; anf dem Wolfensberg ob Veltheim; Brühlberg ob dem Gütsch; auf dem Ebnet ob Töss; Lindberg im Tössertobel; im Brühlbachtobel ob Senuhof.

$R$. cresius L. $\times R$. thyrsanthus sens. coll.

Exsiec. Nr. 255.

Lindberg: hinter dem Büumli.

R. ccesius L. $\times$ R. sulcatus Vest.

Exsice. $\mathrm{Nl} \cdot 256-256 \mathrm{f}$.

Brühlberg-Winterthur ob den Gütschwiesen am Waldrande; Eschenberg gegen das Bruderhaus; Lindberg: am Waldrand beim Bäumli; Wolfensberg' bei Veltheim; Brühlbachtobel ob Sennhof; am Waldrande bei Attikon.

\section{Fragaria L.}

251. F. elatior Ehrh.

Exsice. Nr. 257.

Nicht sehr verbreitet.

Breite häufig (Siegfried, Keller); an der Strasse zum Bäumli (Keller); Wolfensberg (Hug, Siegfried). 
252. F. vesca L.

Exsicc. Nr. 258.

Ueberall.

253. $F$. collina Ehrh.

Exsice. Nr. 259.

Selten.

Südabhang vom Brühlberg (Siegfried); Lindlberg (Hug).

\section{Comarum L.}

254. C. palustre L.

Sümpfe; selten.

Hettlinger Ried (Steiner); Mooshurg bei Effretikon (Caflisch).

\section{Potentilla L.*)}

255. P. erecta L.

Exsice. Nr. 260-260 d.

Wülder ; häufig.

2906. P. strictissima Zimm.

Exsice. $\mathrm{Nr}_{\mathrm{r}} \cdot 261-261 \mathrm{c}$.

Am Süidabhang von Hoh-IVülflingen (Siegfiried); Beerenberg (Siegfried); Wolfensberg (Siegfried); Holzschliage ob Seen am Wege nach Sennhof (Keller); am Tössberg in Uebergängen zur typischen $P$. erecta L. (Keller).

257. P. sciaphila Zimm.

Exsice. Nr. 262.

Eimmal über der Breite (Siegfried); Kemptbrücke (Siegfried); im Walkeweiher (Keller').

258. P. dacica Borbás.

Exsice. Nr. 263, 263 a.

Südabliang des Brühlberg (Siegfried); an Wolfensberw, hier auch mit Uebergiingen zur typischen I'. erecta L. und falla.x Moris (Siegfried).

*) Nachfolgenden Standortsangaben liegen in wesentlichen die vielen Beobachtungen Herrn H. Siegfrieds zu Grunde. In der $\Lambda n-$ ordnmug' und Nomenklatur folge ieh im allgemeinen ' $\mathrm{i}$ immeters Thersicht: „Die europäischen Arten der Gattung Potentilla." Der grösscre 'Teil der von mir um Winterthur gesammelten Potentillen wurde seiner Zeit von Herm Prof. Zimmeter revidiert. Ich spreche ihm anch an dieser Stelle meinen besten Dank aus. 
259). P. fallax Moris.

Exsice. $\mathrm{Nr}$. 264.

Esehenberg (Imloot); Wolfensberwa (sicgtried).

1. reptans L. $\times P$. erecta L.

P. adscendens Gremli $=$ P. Gremlii Zimm.

Exsice. $\mathrm{Nr} \cdot 265$.

Eschenberg' (Hirzel, Imhoof); iiber der Breite massenhaft, ebenso links von der Strasse von der Breite zum Bruderhaus in Strassengraben (Siegfried); links an der Strasse vom Bruderhaus zum Eschenberg (Siegfried). - Die Formen der Eschenberger Standorte neigen in allgemeinen zu $P$. reptans L. Wolfensberg links und rechts der Strasse bei den ersten über Veltheim gelegenen lichten Fichtenbeständen (Siegfried, Keller). Diese Formen neigen mchr zu $P$. erecta L. hin. In Eschenberg f. aprica, Wolfensberg f. umbrosa.

260. P. reptans L.

Exsicc. Nr. 266, 266 i.

Gräben, Wegränder; ïberall.

var. I' microphylla Tratt.

Exsice. $\mathrm{Nl}_{1}, 267$.

Strassenkies hinter der Gastiubrik (Niegfried); Eschenberg mittlere strasse über der Breite dureh den IVald (Siegfried).

261. 1. Anserina L.

Exsice. Nr. 208, 268:

Wege, strassengräben; ïbraall.

f. aurantiaca Zimm.

Bei der Breite rechts an der Strasse gegen VogelsangWinterthur (Siegfried).

f. sericea Hayne.

Exsice. Nr. 269.

Nichit selten.

Schön ausgeprägt auf der Strasse ob dem Haldengut (Keller); auf' der Winterberger Steig (Keller); in Tobel gegen dic Hinterhub (Keller); Wolfensberg (Siegfried). 
f. viridis Koch.

Exsiec. $\mathrm{N}_{1}$. 270 .

Wolfensberg (Siegfried).

26.). I’. mubns Crant\%.

Exsice. $\mathrm{Nr}, 271$.

Sonnige Hügel; häufig.

f. anrantiaca.

Exsice. $\mathrm{Nr}, 272$.

Haldenberg bei Hard-Neftenbach (Keller).

I'. supermbens Cr. $\times I$ '. opaca L. non auct. = P. Kellerii Siegfi.

Exsice. Nr. 273.

Sehr selten.

Etwas unterhalb vom Hard rechts von der Strasse (sicgfried, Keller).

263. P. opaca L. non aluet.

Exsiee. $\mathrm{Nr}$. 274-2741.

Häufig.

264. P. serotina Vill.

Exsice. Nr. 275-275d.

Veltheim bei der Spritzentrotte (Sicufried): zwisehen Wülflingen und Hard am Strassenbord (Siegfried, Keller); in einer grossblütigen Form beim Anental (Keller, Siegtried).

265. I'. Neumamiana Rehb.

Exsice. Nr. 276.

Von der Wirtschaft oberhall, der gerleckten Brïcke bei Pfungen gegen die Rotfarbe links an der Strasse (Siegfried, Keller).

266. I'. longifrons Borbás.

Exsice. Nr. 277.

Am Baeh bei der Rotfarbe und an Abhang der Kiesgrube über der Ziegelei Neftenbach (Siegfried).

267. I'. Siegfriedii Zimm.

Exsice. Nr. 278.

Am Bache bei rler Rotfarbe Neftenbach (Siegfricd). 
268. P. restiva Hall. fil.

Exsiec. Nr. 279.

Nicht ganz typisch am Eulachufer in der Schützenwiese bei Winterthur (Siegfried, Hug).

269. P. Amansiana F. Schultz.

Exsicc. Nr. 280.

Rotfarbe Neftenbach und uiber der Ziegelei Neftenbach gegen die Rotfarbe (Siegfried).

270. P. Billoti N. Boulay.

Exsice. Nr. 281-281 e.

Hoh-Wülflingen.

Typisch auf Hoh-Wülflingen und beim Schweikhof gegen Neuburg am strassenabhang über dem Weihentaler Ried (Siegfried).

271. P. Vitodurensis siegfr.

Exsice. Nr. 282.

Hard unweit des letzten Hauses rechts an der Strasse nach Pfungen an Bord (Siegfried, Keller); am Abhang von der Kiesgrube bei der Ziegelei Neftenbach (Siegfried); Hoh-Wülflingen (Siegfried).

272. P. albescens Opiz.

Exsice. Nr. 283.

In einer nicht ganz typischen Form an Wolfensberg (Keller).

273. P. aumlenta Gremli.

Exsice. Nr. 284, 284 a, 284 b.

Wolfensberg (Siegfried); Hoh-Wülflingen (Siegfried); Hard vor Neftenbach (Siegfried, Keller); Abhänge der Kiesgrube bei der Ziegelei Neftenbach (Siegfried); zwisehen Wartgut und Dättlikon nahe beim Wartgut rechts von der Strasse und rechts am Fussweg zum Wartbad (Siegfried).

274. P. explanata Zimm. = P. prostrata Gremli.

Exsice. Nr. 285.

Au Abhang gegen die Fabrik in Hard; Kiesstellen bei der Ziegelei Neftenbach (Siegfried). 
275. P. intricata Gremli.

Exsiec. Nr. 286.

Bei der Ziegelei Neftenbach gegen die Rottarbe (Siegfried); Auental (Siegfried); in Hard (Siegfried).

276. P. Turicensis Siegfi.

Exsice. Nr. 287-287d.

Am südlichen Bord bei der Kiestrube Neftenbach und rechts vom strïissehen von der Kiesgrube Neftenbach zur' Ziegelei (Siegfried, Keller); Strassenbord bein Wartgut (Siegfried): obere Hub an dem Strassenablang rechts (Siegfried); nieht ganz typiseh in einem Holzsehlag ob Seen an Waldweg nach Semuhof (Keller); ob den Weinbergen von Reutlingen (Keller); Maienried-Wülflingen (Siegfried).

277. P. subopaca Zimm. = P. superopaca L. non auct. $\Varangle P$. rubens Crantz.

Exsice. Nr. 288 .

Zwischen Wülflingeu und dem ersten Hause im Hard (Siegfried); Auental (Siegfried, Keller); am Haldenberg beim Hard-Neftenbach (Keller, Siegfried); Hoh-IVülflingen (Siegfried).

278. P. steritis L.

Exsice. Nr. 289-289 b.

Waldränder, Raine; häutig.

Geum L.

279. G. urbanum L.

Exsice. Nr. 290.

Hecken, lichte Waldstell'n; hiäutig.

280. G. rivale $\mathrm{L}$.

Exsicc. Nr. 291.

An Bäichen, in nassen Wiesen; hä̈nfig.

Anmerkung. Ueber teratologische Abweichungen der Blütenbildung vergl. Keller: Bildungsabweichmogen der Blüten angiospermer Pfimzen im Botan. Centralblatt, Bd. XXXII. 1898. 
Rosa :

281. R. cinnamomea L.

Exsice. Nr. 292.

Wälder um Winterthur": selten.

Brühlberg am Waldıande ob dem Schlosshof auch Steiner und Schellenbaum geben als Standort den Brühlberg an); Lindberg an der Strasse vom Rosenberg zum Walkeweiher.

Zahlreiche Büsche an der Töss ob dem Hard mit gefïllten Blïten, also Gartenfliichtlinge, stellen dic

f. focundissmu Koch dar. Ebenso die Hecke hinter dem Gasthof zum Hirschen in Kyburg.

Exsice. Nr. 293, 293 a.

282. R. alpina L.

Exsice. Nr. $294-294 \mathrm{w}$.

Die Individuen starker Hispiditait werden als f. pyrenaica Chr. bezeichnet; jene, deren Blïtenstiele und Receptakel keine SticIdriisen besitzen als f. levis Chr. Da beide Formen durch zahlreiche Uebergänge nit einander verbunden werden, sind sie unserem Datürhalten nach nur individuelle Modificationen. IVir fülıren sie desshalb nicht besonders anf. Das gleiche gilt für die sog. var. lagenaria Vill. - Individuen mit langen, flaschenförmigen Frïchten und var. globosa Dev. - Individuen mit kugeligen Friichten. Alle diese Modificationen kommen in Gebiete vor.

Eschenberg nicht häufig im Steinbachtobel; um Sennhof reichlich, so im Briilılbachtobel, am Kyburger Rain, Tugsteinhalde, Biliker Tobel; Seemer Rüti bei Kollbrunu; Tobel bei Rykon; Röhrlitobel bei Schlatt; Fuchsloch bei der Winterberger Steig: im Homelholz bei Kyburg; Rossstall ob Kollbrumn.

Anmerkung. In meiner eitierten Arbeit über die zürcherischen Rosen ist eine f. latifolia Seringe aus dem Brïhlbachtobel erwähnt. Fortgesetzte Beobachtungen liessen in ihr eine typische $R$. alpina erkennen, deren Blatter in den letzten Jahren in ilner Mehrheit nicht über die Durchschnittsgrösse der Alpinablätter hinausgiellgell.

*) Die nach folgenden Standortsangaben fiussen tast ausschliesslich ant den Beobachtungen des Verfassers. Die Anordnung folgt der Monographie Christs: Die Rosen der Sthweiz. Vergleiche auch Dr. Robert Kellers Wilde Rosen des Kantons Zürich im Botanischen Centralblatt, Bil. XXXV, 188s. 
li. alpina L. X li. tomentosa sm. forma: loc. cit. R. alpina $\times$ l. mollis Sm.

Exsice. $\mathrm{N}_{1} \cdot 095-2951$.

Brühllachtobel ob Sembof in einer griassern Kall rom strainchern, die bahl mehr der ersten Art, bald mohr lor zweiten sich näliern.

Bezüglich der Fornen verweisen wir anf die citierte Arheit, so wie ant cinen in Vorbereinug begriffenen $\lambda$ rtikel meiner Bejtraige zur schweiz. Phanerogamenflora im Botan. Centralblatt.

29:3. R. pomifera Herru.

Exsice. Nr. 296.

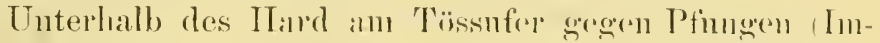
loof ).

2is4. R. tomentosa sin.

Verbieitet.

f. typrica Christ.

Hxsice. Nr. 297, 207 :

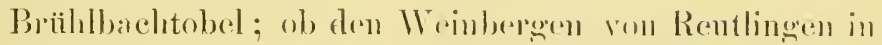
nicht ganz typischer Form.

f. subglobosa Baker.

Exsiec. Nr. :298-298i.

In dieser durch die kugeligen Friiehte gekemmanchucten Modification ist R. tomentosa Sm. durch das gamze Gebiet verbreitet nnd stellenweise häufig

Brahalde-Hünikon; Kyburg am Waldrand links ron der Strasse nach Fehraltorf; an der Strasse von Sichlatt nach Langenhard; Briihlbachtobel; Eschenberg: am Fussweg zum Bruderhaus oberhall, der Breite; zwisehen Nussberon sund Schlatt am Ausgang des Waldes vor Sichlatt; Tobel zur Hinterhub. In einer schïnen, der fo decolorans sich nähernden Form (2098) hinter dem Bïumli in rrsten Sehlag.

f. pseudocuspidata Crépin.

Exsice. Nr. 299.

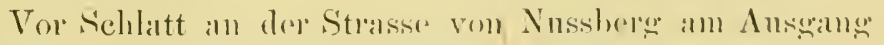
des Tialies. 
f. anthracitica Chr.

Exsice. Nr. 300.

Ob den Reben bei Zïnikon; nicht ganz typisch.

285. R. mubiginosa L.

f. umbellata Chr.

Exsicc. Nr. 301, 301 a, 301 b.

Nicht verbreitet und nur in wenigen nicht ganz ty pischen Sträinchern.

Im Griit bei Dynhard cin Strauch, ähnlich ob der Schwärzi bei Hettlingen; als kleiner Strauch, der den Uebergang zur forma comosa bildet, im Paradies bei OberEmbrach.

f. apricorum Ripart.

Exsice. Nr. 302.

Solten.

In einer Waldwiese bei Schottikon.

f. comosa Chr.

Exsiec. Nr. $303-303 \mathrm{~g}$.

Haiufig.

In Paradies bei Ober-Embrach; am Geltenbühl bei Dittlikon; an der Strasse von Nussberg nach Schlatt kurz vor dem Ende des Waldes; beim Steinbruch ob den Weinbergen von Reutlingen; Ablänge bei der Station Hettlingen; ob der Schwärzi an der Strasse nach Henggart und am Waldrande gegen Hünikon; unterhalb Hoh-Wülflingen; bei Kyburg; Reben ob der Rotfarbe Neftenbach.

f. Gremlii Chr.

Exsiec. Nr. 304-304 c.

Ob den Weinbergen ron Rentlingen mehrere Strïiucher.

2Rti. R. agestris Sav.

Nicht häufig.

f. pubescens Rapin.

Exsice. Nr. 305, 305 a.

Ander Kyburger Schlosshalde; bei der Station Thalheim. 
287. R. tomentella Léman.

Nicht häufig.

f. typica Chr.

Exsice. Nr. 306-306 d.

Weinberge ob Reutlingen; Hünikon: typisch und in etwas abweichenden Modifieationen an der Brahalde.

f. concinna Chr.

Im Gebicte nicht ganz identisch mit Christ's forma. Exsiec. Nr. 307, 307 i.

Sehottikon; im Paradies bei Ober-Embrach.

f. affinis Chr.

Exsice. Nr. 308, 308 i.

An der Strasse von Nussberg nach Schlatt.

288. R. trachyphylla Ran.

Exsice. Nr. 309-309 e.

f. typica Christ.

Im Kramer bei Hoh-Wülflingen; oberhalb Neuburg; im Kapf anf dem Brühlberg ziemlich häutig; an Geltenbühl bei Dättlikon; in Bähntal an der Strasse nach Nussberg; im Türliacker in Ober-Langenhard; am Wegrom Kollbrumn nach Ober-Langenhard.

289. R. canina L.

f. Lutetiana Chr.

Exsice. Nr. 310-310h.

Durch das ganze Gebiet meist nicht selten.

Lindberg; Wolfensberg; Brïlllberg; in Brühlbachtobel ol) Semulıof; Kylurrg; Kämleten; in der Eigelhart bei Pfungen; in Paradies bei Ober-Embrach; Bruitten; Tobel zur Hinterhub; Reben ob der Rotfarb Neftenbach; Bolster'nbuck bei Kollbrum. In Bezug auf Form und (riö̈sse dere Blitter und der Receptalkel vidfach indernd.

f. dumalis Chr.

Exsice. Nr. 311-311 i.

Durelı dals gannze Gebiet nicht seltern. 
Beim Walkeweiher: Wolfensberg ob Veltheim; Brühlberg bei Winterthur; im Gütseh; im Kapf; im Schönbühl beim Ruchegg: ob den Weinbergen ron Reutlingen; ror Dättlikon; bei der Schwairzi-Huttlingen; Winterberg; Tugsteinhalde Somnhof; Briitten; Obre-Embrach; Elsan; 'Tobel zur Hinterhub.

f. bisèrrata Baker.

Exsice. $\mathrm{Nr} .312-312 \mathrm{l}$.

Dureh das ganze Gebiet nicht selten.

Lindberg; Wolfensberg; Hoh-Wülflingen; Brühlberg; Weinberge ob Reutlingen; Tierlisberg ob Kollbrumn; im Siiftholz bei Nussberg; zwischen Schlatt und Langenhard; Ober-Embrach; ob Kempttal gegen Winterberg; an der Steig nach Brütten; vor Daittlikon; 'Tobel zur Hinterhub; Bolsterenbuck bei Kollbrum.

f. Andegavensis Rapin.

Exsice. Nr. 313-31:3d.

Nicht häufig.

Brïhllberg, jedoch nieht ganz typiselı; 'T'̈̈ssberg' : anf dem Ebnet; ob Kempttal: Brïhlbachtobel.

f. hirtella Christ.

Exsice. $\mathrm{Nr}$. 314-314e.

Hoh-Wülflingen; an Tössber's; an lrehel zwisehen dem Wartgut und Daittlikon; zwischen Nussberg nud Sehlatt. f. certicillacantha Baker.

Exsice. Nr. 315-315е с.

An der Fahrstrasse zum Giitsch bei Winterthur: Geltenbühl bei Dättlikon; Weinberger bei Hünikon; im Griit bei Dynhard.

f. hispidula Ripart.

Exsice. $\mathrm{Nr} .316-316 \mathrm{c}$.

Wolfensberg ob den Veltheimer Weinbergen; Waldrand beim Paradies Ober-Embrach; Waldrand links an der Strasse Elsau-Schottikon.

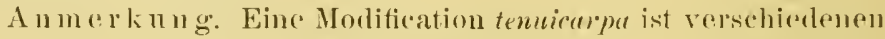
der vorgenamiten Formen muterynorinen. 
290. R. glanca Vill.

f. typica Chr.

Exsice. Nr. 317.

Auf dem Wolfensberg; an der Töss ob dem Hasrd; Briihlbachtobel ob Sennhof.

f. complicata Chr.

Exsicc. Nr. 318.

Am Wolfbühl ob der Hofstatt unterhalb Hoh-W Wülflingen.

f. subcannia Chr.

Exsice. Nr. 319-319d.

Lindberg; am Waldrand ob dem Süsenberg; beim Bïmmli; im Kapf auf dem Briuhllberg.

f. pilosula Chr.

Exsiec. Nr. 320.

Wolfensberg; im Kiıf am Briilılberg.

R. ferruginea Vill.

Hirzel in Köll. Phanerog.

Sehr fraglich oder doch nur Gartenfliichtling!

291. R. dumetorum Th.

f. urbica Lehm.

Exsiec. Nr. 321, 321 a.

Am Tössberg; Kämleten; am Züniker Kirehweg; bei der Nagelfuhbank zwischen Elsan und Schottikon.

f. platyphylla Chr.

Exsicc. 322-322 h.

Brühl: iiber den Reben rom Grafenstein; Wolfensberg; an der Strasse zwisehen Nussberg und Schlatt: an der Züniker Halde; am Weg von Madlikon nach Ober-Embrach ; Breite bei Brütten; im Paradies bei Ober-Embrach; zwischen Kempttal und Winterberg; bei Kiimleten; Itinilkon.

f. Thuilleri Chr.

Exsice. Nr. $323-323 \mathrm{~d}$.

Hoh-Wülflingen; an Waldrande ol, Schottikon: im Paradies bei Ober-Embrach: Haldenberg bei Dittlikon: (ieltenbiihl bei Diittlikon; in der lisgellart bei Pfungen. 
f. trichoneura Chr.

Exsice. Nr. 324-324 c.

Weinberge zwischen Stadel und Ruehegg; Kyburg beim Aerni-Haus; im Paradies bei Ober-Embrach; ab dem Grüt bei Dynluard.

f. obtusifolia Chr.

Exsice. Nr. 325, 325 a.

Im Hohlweg am Goldenberg bei Winterthmr; Wurmetshalde bei Dittlikon.

f. Deseglisei Chr.

Exsice. $326-326$ h.

Heeken beim neuen Kirchlof in Winterthur; am Waldrand des Eschenberg ob dem Gut; auf dem Wolfensberg; Brïhlbachtobel ob Semnhof; Kämleten; am Wege von Schottikon nach Elsau; Grüt bei Dynhard; zwischen Station Thallıeim und Griit; am Kyburger Sehlossrain.

f. pseudocollina Chr.

Exsice. Nr. 327, 327 a.

Nicht typisch und stets gegen die f. Deseglisei neigend.

Im Grüt bei Dynhard; zwischen Elsau und Rïtersehen.

292. R. coriifolia Fries.

f. scaphusiensis Chr.

Exsice. $\mathrm{Nr} .328$.

Wolfensberg ob den ersten Häusern ron Wülflingen. f. subcollina Chr.

Exsice. Nr. 329, 329 a, 329 b.

Brühlbachtobel ob Sennhof; Winterberg; Tössberg.

R. corifolia Fr. $\times$ R. gallica.

Exsicc. Nr. 3:30, 330 a, 330 b.

An der Fahıstrasse zum Gütseh bei Winterthur; bein Schloss Mörsburg (spontan?).

293. R. arvensis $\mathrm{L}$.

f. umbellata Godet.

Exsice. Nr. 331, 331 a.

Wolfensberg; Hoh-Wüilflingen. 
f. repens Chr.

Exsice. Nr. 332, 332 a.

Durch das ganze Gebiet sehr häufig.

Lindberg; Wolfensberg; Tössberg; Hoh-Wülflingen ;

Eschenberg; Bruilhlbachtobel; Tobel zur Ininterlub; Winterberger Steig; ob der Station Seen; Bolsterenbuck bei Kollbrumn.

\section{Agrimonia L.}

294. A. Eupatoria L.

Exsice. Nr. 333.

Waldränder, Abli:inge; häufig.

Alchemilla Scop.

295. A. vulgaris $\mathrm{L}$.

Exsice. Nr. 334.

Waldränder ete.; hïufig.

296. A. arvensis $\mathrm{L}$.

Accker; selten.

Un Winterthur (Steiner).

Poterium L.

297. P. dictyocarpum Sp.

Exsice. Nr. 335.

Wiesen, Wegräinder etc.; gemein.

Sanguisorba L.

298. S. officinalis $\mathrm{L}$.

Exsice. Nr. 336.

Ruchried bei der Kreuzstrasse (Siegfried).

Pirus L.

299. P. Malus L.

Exsice. Nr. 337.

Wälder; hin und wieder.

Wolfensberg (Siegfried); Kyburger S'chlosshalde(K'eller); um Ober-Embrach (Keller).

300. P. communis L.

Exsice. Nr. 338.

Wilder; hin und wieder.

Wolfensberg (Siegfried); Eigellant bei Pfungen (Keller). 


\section{Sorbus L.}

301. S. Aucuparia L.

Exsice. Nr. 339.

Wälder; häinfig.

302. S. Aria $\mathrm{Cr}$.

Exsice. Nr. 340.

Wälder; häufig.

S. Aria Cr. $\times$ torminalis $\mathrm{Cr}$.

Exsice. Nr. 341, 341 a.

Züniker Halde (Hirzel): 'Tössberg an der Nordseite iiber dem Bahnwiirterhäusehen an der Waldshnter-Linie (Siegfricd); am Waldrand zwischen Dittnau und Neuburg (Siegfried); Alt-Wülfingen (Jäggi).

303. S. torminalis $\mathrm{Cr}$.

Exsice. Nr. 342, 342 a.

Wälder; häufig.

Mespilus Lan.

$M$. germanica $\mathrm{L}$.

Hirzel in Köll. Phanerog.

Cratægus L.

304. C. oxyacantha L.

Exsice. Nr. 343.

Heeken, Wälder; häufig.

305. C. monogyna Jug.

Exsiec. Nr. 344.

Wälder; nicht so häufig wie vorige.

Brïllbachtobel (Keller); Kylurrg (Schellenbaum).

Cotoneaster Med.

306. C. tomentosa Lindl.

Exsicc. Nr. 345.

Wolfensberg (Herter, Hug, Siegfried); Tössberg vor Hoh-Wülflingen (Weinmann, Siegfried, Hug, Herter, Keller); Ebnet (Cafliseh); Brühlbachtobel ob Sennhof (Keller); Kylunger Schlossrain (Keller); Alt-Wülflingen (Catlisch). 
XxVII. Philadelphea Don.

\section{Philadelphus L.}

307. Ph. coronarius L.

Exsice. Nr. 346.

Hin und wieder verwildert.

An der Kyburger Schlosshalde.

XXVIII. Onagrarieat Juss.

\section{Epilobium L.*)}

308. E. angustifolium L.

Exsice. Nr. 347, 347 a.

Holzschläge; gesellig und verbreitet.

Eschenberg (Hug); über der Breite; an der Waldstrasse über Seen gegen den Eschenberghof; an der Töss; auf dem Brühllberg (Keller); Waldschliage ob Wiesendangen (Keller).

309. E. rosmarinifolium $\mathrm{Hk}$.

Exsice. Nr. 348, 348 a.

Kiesige Stellen; nicht häufig.

Wolfensberg; an den Ufern der Töss unterhalb Wülflingen (Stciner, Imhoof); unterhalb der Station Pfungen beim Strasseneinsehnitt (Keller); Pfungen (Catliseh); linkes Tössufer zwisehen Bodmersmuihle und Hard.

310. E. hirsutum L.

Exsicc. Nr. 349-349 c.

Gräben; verbreitet.

Eschenberg; Eulaeh; Bettwiesenrierl; Kyburw (Keller); ob dem Gütsch (Keller); Hettlingen (Hug).

f. putate Haussk.

Exsice. Nr. 350.

Winterthur, im Strassengraben östlich neben dem Spital.

*) Nacholgende Standortsiibersicht der $\Lambda$ rten und Formen ("ntnehme ich, wo nichts anderes angegeben ist, den Vitteilungen meines Freundes H. Siegefried, dem ieh anch cine grö̈ssere Zahl ron l'rofessor Ha us skech trevidierter Epilobienexsiccaten verdanke. 


\section{f. adenocarpa lanceolata Haussk. \\ Bettwiesenried.}

311. E. parviflorum Schreb.

Exsice. Nr. 351-351 c.

Gräben, Wälder; überall.

f. verticillatum Haussk.

$\mathrm{Ob}$ der Breite.

f. aprica Haussk.

Exsicc. Nr. 352.

Trockene, sonnige Waldschläge.

f. umbrosa Hanssk.

Exsicc. Nr. 353.

Hinter der Gasfabrik im Strassengraben; Rosenberg links an der Strasse in Gräben.

f. umbrosa aquatica Hanssk.

Hinter der Gasfabrik.

E. parviflorum Schreb $\times$ E. roseum Schreb $=$ E. opacum Peterm.

Exsicc. Nr. 354 .

Hinter der Gasfabrik; Strassengraben beim HaldengutWinterthur und beim Kantonsspital; Strassengräben beim Rosenberg; Bettwiesenried.

E. parviflorum Schreb $\times$ E. roseum Schreb $f$. putata Haussk. Bettwiesenried; im grossen Torfloch beim Rosenberg. E. superparifforum Schr. $\times$ E. roseum Schr. Haussk.

Links im Strassengraben uiber Veltheim zum Wolfensberg.

E. parviforum Schr. $\times$ E. montanum L. $=$ limosum Schr. Ueber der Breite.

312. E. tetragonum L.

Exsice. Nr. 35̃5.

Sehr spärlich über der Breite-Winterthur; bei der Brücke über die Eulach oberhalb der Festhütte. 
E. udnatum Grsh. × E. Lamyi F. S'z.

Ueber der Breite ein Exemplar.

313. E. Lamyi F. Sz.

Exsice. Nr. 356.

Nicht selten in der Ungebung von Winterthur.

Ueber der Breite in den Schlägen; in den Waldschlägen am Fussweg zwischen dem Eschenberghof und Linsetal; Schläge ob dem Gütli-Lindberys.

f. umbrosa.

Eschenberg' über der Breite.

f. ammua.

Eschenberg über der Breite.

f. hiemis.

subf. microphylla.

Eschenberg über der Breite.

E. Lamyi F. Sz. $\times$ E. parviflorum śchr. = E. Haussknechtianum Borb.

Exsice. Nr. 357.

Hinter der Gasfabrik; Eschenberg über der Breite.

E. Lamyi F. Sz. × E. montanum L.

Ueber der Breite.

314. E. obscurum Schreb.

Ueber der Breite sehr reichlich.

f. verticillata.

Ueber der Breite.

f. annua.

Ein Exemplar über der Breite.

E. obscurum Schr. $\times$ E. montamum L. = E. agginegatum Cr.

Waldschläge über der Breite in Esehenberg.

315. E. roseum Schr.

Exsicc. $\mathrm{Nr}$. 358, 3528 a, 358 b.

Häufig.

f. putata Haussk.

Hinter der Gasfabrik. 
f. umbrosa.

Hinter der Gastabrik und am Strassengraben beim Haldengut-TVinterthur.

f. aprica Haussk.

Strassengraben bei der Brauerei Haldengut.

316. E. montanum L.

Exsice. $\mathrm{Nr} .359$.

Sehr häufig.

f. verticillata Haussk.

Sehr selten. Ueber der Breite in zwei Exemplaren.

f. minor Haussk.

Exsice. Nr. 360.

Als Modif. aprica et umbrosa überall in Waldschlägen mit der typischen For'm.

f. umbrosa Haussk.

Exsice. Nr. 361.

Mit der typischen Form.

E. montanum L. $\times$ E. parviflorum Schreb.

Exsicc. Nr. 362.

Hinter der Gasfabrik; über der Breite.

317. E. palustre I.

Exsicc. Nr. 3633.

Hettling'er Ried (Hir'zel).

\section{Oenothera.}

318. O. biennis.

Exsice. Nr. 364.

Flussufer, Eisenbahmdämme; häufig.

Vogelsang (Siegfried); Linsetal (Keller, Herter); Eisenbahndamm vor Kempttal (Keller); an der Töss (Herter, Steiner); zwischen Kollbrumn und Weisslingen (Hug).

Circæa L.

319. C. Iutetiana L.

Exsicc. $\mathrm{Nr}_{1} .365$.

Wälder; gemein. 
XXIX. Haloragea Br.

Hippuris L.

320. H. vulgaris L.

Teiche; selten.

Kyburg (Ziegler).

Myriophyllum L.

321. M. certicillatum L.

Exsice. Nr. 366, 360 a, 366 b.

In Teichen, Riedbaichen, Grïben: nicht selten.

Hettlinger Ried (Hirzel, Hng, Siegfiried); Wiescndangond Ried (Hirzel); Feuerweiher Ohringen (Siegfried, Herter); Ricdbach vor Ohringen (Keller); Torflöcher im Weiher'tales Ried (Siegfried, Her'ter); Ruchried (Siegfiried).

\section{Callitrichinea Lk.}

\section{Callitriche L.}

322. C. stagnalis Scop.

Exsicc. Nr. 367.

Am Hinterendliker Waldrand oberhall, der Hirschen wiese in einem Sumptgraben (Hirzel): Ohringer Fencrweilıer (Siegfried, Herter').

f. platycarpa Kütz.

Ohringer Fenerweiher (Siegfried).

323. C. vernalis $\mathrm{K}$.

Exsice. Nr. 368.

Ohringer Fenerweiher (Siegfried, Herter); Kylumgen ('Ziegler); Gräben an der Eulach (Steiner).

324. C. hamulate K.

Ohringer Fenerweiher (siegfried).

XXXI. Ceratophyllea Gray.

\section{Ceratophyllum L.}

325. C. demersum L.

Exsice. Nr. 369.

Ohringer Fenerwalles (siegtried); Tortlizher in Ruehried (Siegfried); WVeihertaler Ried (Siegfried). 
XXXII. Lythrariea Juss.

Lythrum L.

326. L. Salicaria L.

Exsice. Nr. 370.

Gräben, Sumpfwiesen; überall.

XXXII. Tamariscinent Desv.

Myricaria Desv.

327. M. germanica Desv.

Exsice. Nr. 371.

Flussufer; nicht häufig.

An der Töss (Weimmamn, Herter'); an der 'Töss unterhalb des Hard (Steiner, Imhoof); an der Töss beim Reitplatz (Gamper); gegen Kyburg (Steiner).

Herniaria L.

Paronychiea St. Hil.

H. ylabra L.

Steiner in Köll. Phanerog.

XxxIV. Portulacacea Dc.

Portulaca L.

328. P. oleracea L.

Exsice. Nr. 372.

Wege, Schuttstellen; nicht hïutig.

Beim Rychenberg-IVinterthur (Keller); beim alten Turnhauts an der Trollstrasse (Keller).

\section{Scleranthea Lk.}

Scleranthus L.

329. Sc. annus $\mathrm{L}$.

Exsice. Nr. 373, 373 a.

Auf Stoppelfeldern hin und wieder.

XXXY. Crassulacere De.

Sedum.

330. S. maximum Sut.

Exsice. Nr. 374. 


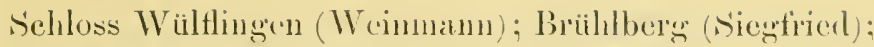
an der Waldstrasse vom Eschenberghof nach Seen (Sivgfried).

S. vulgare Lk.

Effretikon (Caflisch).

331. S. rupestre L.

Exsice. Nr. 375.

Unterhalb Wülttingen gegen dals Hard (Steiner, Weinmann, Schellenbaum, Herter, Siegfried).

332. S. sexangulare L.

Exsice. Nr. 376, 376 a.

An Mauern, kiesigen Stellen; ziemlich häutig.

Hinter der Gasfabrik (Siegfried, Keller); Semhof' (Keller); Wolfensberg (Herter); unterhalb Wülflingen beim Hardberg neben S. rupestre (Herter).

333. S. acre L.

Exsice. Nr. 377.

Sonnige Weiden längs der Töss zwisehen Wülflingen und dem Hard (Schellerbaum).

334. S. album L.

Exsice. Nr. 378.

An Mauern.

Wolfensberg (Siegfried).

XXXrII. Saxifragacea De.

\section{Saxifraga L.}

335. S. mutata L.

Exsice. Nr. 379.

Selten.

Brühlbachtobel ob Semnhof (Keller).

336. S. granulata L.

Selten.

Oberhalb Kempttal an dem Balmdamm zwisehen Station und Effretikon (Siegfried). 
337. S. tridactylites L.

Exsice. Nr. 380.

Selten.

Vor Ober-Ohringen (Hirzel).

Chrysosplenium L.

338. Ch. alternifolium $\mathrm{L}$.

Exsice. Nr. 381.

Feuchte, schattige Orte; sehr liäufig.

\section{Umbellate L.}

\section{Laserpitium L.}

339. L. Tatifolium L.

Bergwälder; selten.

Linsetal (Herter).

Orlaya Hfn.

340. O. grandiflora Hfin.

Exsice. Nr. 382.

Aecker; sclten.

Pfungen (Weinmann); Ohringen (Keller).

\section{Daucus L.}

341. D. Carota L.

Exsice. Nr. 383, 383 a.

Wiesen; übcrall.

\section{Caucalis Hfn.}

342. C. daucoides L.

Exsice. Nr. 384.

Aecker; ziemlich häufig.

Turmhalde (Siegfried); Ohringen (Siegfried, Keller); vor Hettlingen (Siegfried); Bruni bei Pfungen (Keller); Acker ob der Turmhalde (Herter).

\section{Torilis G.}

343. T. Anthriscus Gm.

Exsice. $\mathrm{N}_{1}$. 385.

Häufig an Wegräinder'n ete. 
An der Eulach (Siegfried, Herter); hinter der Giesserei (Siegfried); Wolfensberg (Siegfried); Itoh-Wülflingen (Siegfried); Brühlberg (Siegfried); Eschenberge (Hug).

344. T. helvetica Gm.

Exsice. Nr. 386.

Aceker; selten.

Bei Pfungen (Hirzel).

\section{Angelica L.}

345. A. silvestris L.

Exsice. Nr. 387, 387 ஃ.

Fenehte Waldstellen, (iriiben; sehr hinufig.

\section{Selinum L.}

346. S. Carvifolia L.

Hettlinger Ried (Steiner).

\section{Peucedanum L.}

347. P. Cervaria Cuss.

Exsiec. Nr. 388.

Hügel : nieht selten.

Südseite des Tössberges bei Hoh-Wülflingen (Steiner, Jäggi, Siegfried, Hug, Keller, Herter); Wolfensherg (Koller, Herter, Siegfried); um Kyburg (Keller); Beerenberra (Keller, Siegfried); Irehel (Herter).

:34. P. palustre L.

Exsiee. Nr. 389.

Sumpfwiesen; ziemlich verbreitet.

Ohringer und Hettlinger Ried (Hirzel, Steiner, Siegfried); Ruchried (Siegfried); Weihertaler Ried (Siregfried, Herter); Südseite des Tössberges in den Sumpfwiesen bri Neuburg (Siegfried, Keller).

\section{Pastinaca L.}

349. P. sativer L.

Exsice. Nr. 390.

Wirsen, Wegrïnter; semein. 


\section{Heracleum L.}

3.)0. H. Sphondylium L.

Exsicc. Nr. 391.

Wiesen; gemein.

f. elegans Jacq.

Exsice. $\mathrm{Nr} .392$.

Hin und wieder in Bergwiesen.

Wiesen an der Töss nahe der Kemptmündung (Keller); sehr typisch ob Kempttal gegen Winterberg (Keller).

Fæniculum Ad.

351. F. officinale Ad.

Exsice. Nr. 393.

Ungebaute Orte; selten.

Beim Kugelfang in Winterthur (Keller).

Silaus Bess.

352. S. pratensis Bess.

Exsice. Nr. 394.

Riedwiesen.

Totentialchen (Herter) und anderwärts.

Aethusa L.

353. Ae. Cynapium L.

Exsice. Nr. 395.

Wegränder, Stoppelfelder, Aecker; hïufig.

Chærophyllum L.

354. Ch. hirsutum L.

Exsice. Nr. 396.

Gräben, feuchte Wiesen; nicht häufig.

Am Graben in der Turmlalde (Siegfried, Herter); Waldgrïben im Eschenberg (Siegfried).

355. Ch. aureum L.

Exsicc. Nr. 1007.

Auf der Breite in dem Buschwerk nach dem Walde (Siegfried, Hug, Herter).

C\%. temulum Is

Steiner in Köll. Phanerng. 
Anthriscus Hfn.

35) (6. A. silvestris $\mathrm{Hfu}$.

Exsice. Nr. 397.

Wiesen; iiberall.

Scandix L.

357. s. Pecten veneris L.

Exsice. Nr. :398, 398 a, 398 b.

Unter Getreide: nicht selten.

Vor Oberwinterthur und hinter dem Dorfe gegen den Lindberg (Keller); Ohringen (Keller, Siegfried); im Bruni vor Pfungen und in den Aeckern gegenüber vom Wartbad (Keller); Wolfensberg. (Hug, Siegfried); Aecker un Töss (Cafliseh, Siegfried); Aecker im Oberfeld-Wülflingen (Herter).

Sium L.

3\%. s. angustifolium L.

Exsice. Nr. 399.

Gräben, Bäche.

Um Pfungen (Steiner); Hettlingen (Siegfried, Hug): Kreuzstrasse (Siegfried); (rräben in den Riedwiesen zwischen Unter-Ohringen und Aesch zum Teil massenhaft (Keller); Ruchried Ober-Ohringen (Hug, Siegrfried); Strassengraben ob dem Rosenbercr).

Ammi L.

35.9. A. majus L.

Exsice. Nr. 408.

Geiselweid-Winterthur in einem Kleeacker (Sichellenbaum).

\section{Aegopodium L.}

36. A. Podagraria L.

Exsicc. Nr. 400, 400 ¿.

Hecken, Baumgiiten; iiberall.

\section{Pimpinella $\mathrm{L}$.}

361. P. magna L.

Exsice. Nr. 401.

Waldwege, Waldrïnder: sehr häutig. 
362. P. Saxifraga L.

Exsice. $\mathrm{Nr}$. 402.

Trockene Hüigel; häufig.

var. hircina Mch.

Exsice. Nr. 403.

Findet sich in meinem Herbarinm ron Hirzel, jedoeh ohne besondere Standortsangabe.

Carum L.

363. C. Carvi L.

Exsice. Nr. 404.

Wiesen; häufig.

Bupleurum L.

364. B. rotundifolium L.

Exsice. Nr. 405.

An dem Büntenwege, der von der Obermühle ans nach dem Mattenbach führt (Schellenbaum); beim Brïhl (Weinmamu); eimmal als Gartenunkraut in Tïss (Caflisch).

B. falcatum L.

Clairville in Köll. Phanerog:.

Conium L.

C. maculatum L.

Hirzel in Köll. Phanerog.

Sanicula L.

365). S. europaca L.

Exsice. $\mathrm{Nr}_{1}, 406$.

Wälder; sehr hiinfig.

Hydrocotyle L.

36it. H. vulgaris $\mathrm{L}$.

Exsice. Nr. 407.

Nach Hirzel bei "Winterthur": Hettlinger Ried (Steiner).

XXXVIII. Araliacea Juss.

Hedera L.

367. H. Helix -L.

Exsice. Nr. 409.

Wailder; überall. 


\section{Cornete De.}

\section{Cornus L.}

368. C. sanguinea L.

Exsicc. Nr. 410.

Gebüsche, Wälder; hänfig.

369. C. mas L.

Exsice. Nr. 411.

Hecken, gebaut; selten verwildert.

\section{Loranthaces Don.}

\section{Viscum L.}

370. V. album L.

f. platyspermum Keller.

Exsice. vergleiche Belege zu Beiträge der schweizerischen

Phanerogamenflora: II. Die Coniferenmistel im Bot. Centralblatt, 1890.

Bewohner von Laubhölzern: Obstbäume, Schwarzpappel, Hainbuche。

Auf Pirus malus durch das Gebiet immer noch zu häufig; auf Populus nigra im Sennhof (Keller); anf Carpinus Betulus am Kyburger Schlossrain (Keller).

f. hyposphcerospermum Keller.

Modif. latifolia Keller.

Auf Weisstannen; nicht selten.

Eschenberg: an vielen Stellen (Keller); am Kyburger Sehlossrain (Keller).

\section{Caprifoliacea.}

Viburnum L.

371. V. Opulus L.

Exsice. Nr. 412, 412 a.

Gebüsch, Wälder; nicht selten.

Eschenberg: ob dem Gut (Keller); Tr̈̈ssberg (Keller, Siegfried); um Kempttal (Keller); Briihlbachtobel ob Sennhof (Keller). 
372. V. Lantana L.

Exsice. Nr. 413, 413 a.

Wie vorige.

\section{Sambucus L.}

373. S. racemosa L.

Exsice. Nr. 414, 414 a.

Wie vorige.

374. S. nigra L.

Exsice. Nr. 415.

Wie vorige.!

375. S. Ebulus L.

Exsice. Nr. 416.

Wie vorige.

Adoxa L.

376. A. moschatellina L.

Exsice. Nr. 417.

Turmhalde (Imhoof, Weinmann, Steiner, Gamper, Ziegler, Hug, Keller, Siegfried). Auch hier kaum spontan.

\section{Lonicera L.}

377. L. nigra L.

Gebiiseh.

Esehenberg (Herter).

378. L. Yylosteum L.

Exsice. Nr. 418.

Gebüsch, Wälder; überall.

379. L. alpigena $\mathrm{L}$.

Exsice. Nr. 419, 419 a, 419 b.

Bergwälder.

Sennhof (Imhoof); Fussweg zwisehen dem Eschenberghof und Linsetal (Siegfried); reichlich im Brühlbachtobel (Keller); Vogthalde bei Kyburg (Pfau, Ziegler); gegen Kyburg (Steiner). 


\section{Rubiacea Juss.}

\section{Galium L.}

380. G. boreale L.

Exsicc. Nr. 420.

Fenehte Wiesen und Waldstellen.

Am Tössrain (Sehellenbanm); Kyburg (Schellenbaum).

381. G. rotundifolium L.

Exsice. Nr. $421-421 \mathrm{e}$.

Wälder; ziemlich hänfig.

Eschenberg (Schellenbaum, Herter, Hug, Siegfried); Tössberg (Keller); Winterberger Steig (Keller); Eggwald ob Wiesendangen (Keller); Brühlberg (Herter, Siegfried); Hoh-Wülflingen (Cafliseh); Wolfensberg (Siegfried); Lindberg (Siegfried).

382. G. Mollugo L.

Exsiee. $\mathrm{Nr} .422$.

Wegränder, Waldwiesen ete.; sehr häufig.

383. G. sitvaticum L.

Exsicc. $\mathrm{Nr}$. 423, 423 a.

An Waldstrassen nicht selten.

384. G. silvestre Poll.

Exsice. Nr. 424.

Waldränder, Triften.

385. G. uliginosum L.

Exsice. Nr. 425.

Sümpfe; nieht häufig.

Weihertaler Ried (Siegfried); in Eschenberg am Wege gegen den Eschenberghof links von der Strasse (Siegfried).

386. G. palustre L.

Exsiec. Nr. 426, 246 i.

Sumpfwiesen, Gräben; sehr häufig.

387. G. verum L.

Exsice. Nr. 427.

Raine, trockene Wiesen; häutig. 
388. G. tricorne With.

Dättlikon, Irchel (Jäggi).

389. G. Aparine L.

Exsiec. Nr. 428.

Aeeker, Heeken; häufig.

390. G. Cruciata Seop.

Exsice. Nr. 429, 429 a.

Hecken, Gräben ete.; häufig.

Asperula $\mathrm{L}$.

391. A. odorata L.

Exsice. Nr. 430.

Wälder; sehr hänfig.

392. A. cynanchica L.

Exsice. Nr. 431.

Trockene Orte unserer Hügel; sehr häufig.

393. A. arvensis $\mathrm{L}$.

Exsicc. Nr. 432.

Aecker; selten.

Bei Pfungen auf Aeckern beim mittleren Bruni (Hirzel).

\section{Sherardia.}

394. Sh. arvensis L.

Exsiec. Nr. 433.

Aeker; sehr häufig.

\section{Valerianea De.}

Valeriana L.

395. V. officinalis $\mathrm{L}$.

Exsice. Nr. 434, 434 a.

Nicht selten.

Esehenberg (Siegfried); an der Töss (Sehellenbaum, Siegfried, Keller); an der Kempt vor Kempttal (Keller); Bettwiesenried bei Ohringen (Hug, Siegfried); Neuburge r' Ried (Siegfried). 
396. V. sambucifolia Mik.

Exsicc. Nr. 435.

An Bächen, in Wäldern; verbreitet.

Im Linsetal an der Töss zwischen Sennhof und der Kyburger Brüeke (Siegfried, Keller); Eschenberg iiber der Breite (Sicgfried); an der Töss unterhalb Töss (Siegfried); waldiger Abhang gegeniiber vom Kloster in Töss (Siegfried); am Bache vor der Miihle in Weisslingen (Keller)

397. V. dioica L.

Exsice. Nr. 436, 436 i.

Griiben, feuchte Wiesen; überall.

Valerianella Hall.

398. V. Olitoria Pall.

Exsicc. Nr. 437, 437 a.

Aecker; häufig.

399. V. Morisonii K.

Exsice. Nr. 438.

In Getreideäckern nicht selten.

400. V. Auricula De.

Exsice. Nr. 439.

Viel seltener als vorige Arten.

Eschenberghof gegen das Bruderhaus (Siegfried); Kyburg (Pfau).

\section{Dipsacere De.}

Scabiosa L.

401. S. Columbaria L.

Exsice. Nr. 450.

Trockene Wiesen; häufig.

Succisa Mich.

402. S. pratensis Mch.

Exsice. Nr. 451, 451 a.

Feuchte Waldwiesen; häufig. 
Dipsacus L.

403. D. silvestris Huds.

Exsice. Nr. 452, 452 a.

Selten.

An feuchten Stellen des Eschenberg (Imhoof); Kyburger Schlosshalde (Pfau, Keller); im Walde beim Weiher vor Ober-Ohringen (Hug, Siegfried); an der EulachSchützenwiese (Hug, Siegfried).

404. D. pilosus L.

Exsice. Nr. 453.

Auf Schuttstellen; selten.

Einmal an einer Hecke bei der Kirche in Töss (Hirzel).

Trichera Schr.

405. T. arvensis Schrad.

Exsice. Nr. 440.

Wiesen; überall.

406. T. silvatica Schr.

Exsice. Nr. 441.

Wälder; häufig. 



\section{QK 315. Kew York Botanical Garden Library \\ Keller. ${ }^{2} 43$ T.1 pt. Halfte 1 \\ (III Robert/Flora von Winterthur

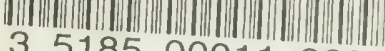 \\ 35185000118602}




\section{Wekes

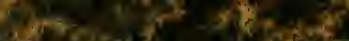

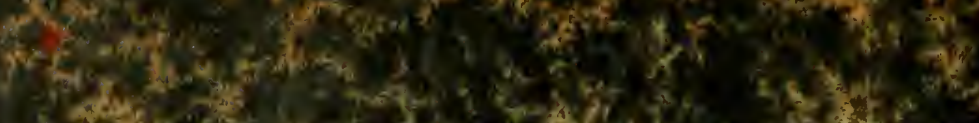

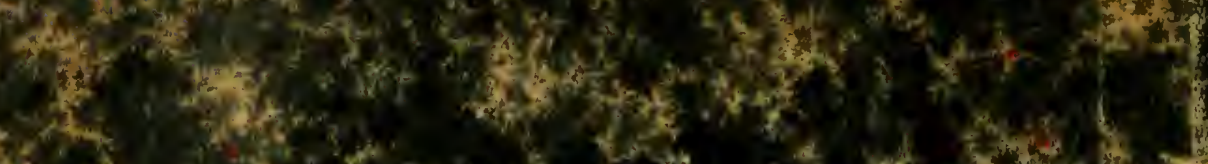

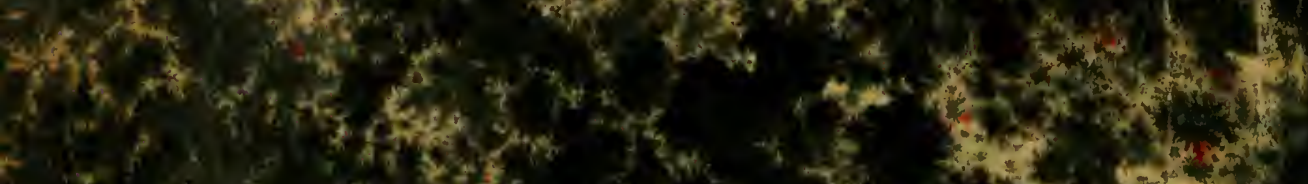

$7 x^{2}+x^{2}+x^{2}+x^{2}$ N. $x$

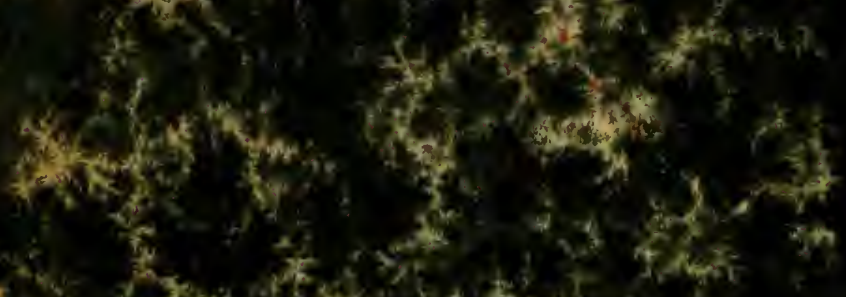

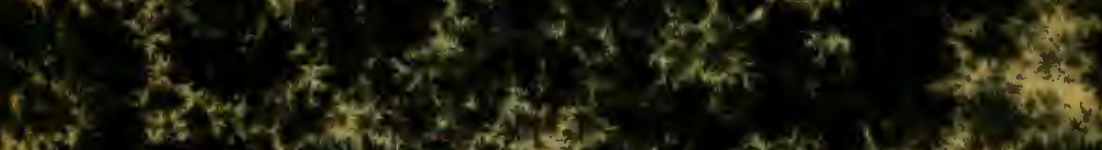

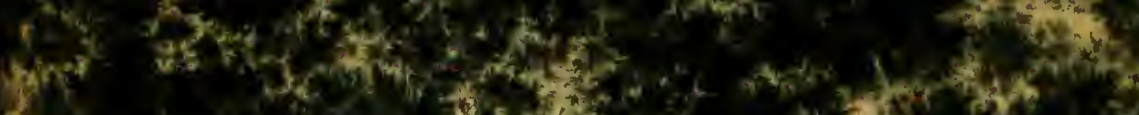

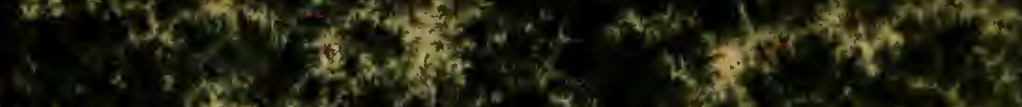
(1)

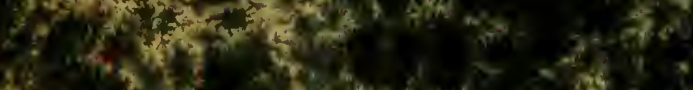

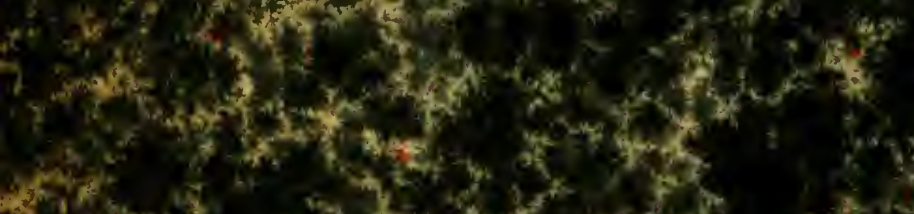

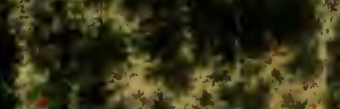

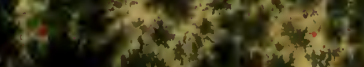
S.r.

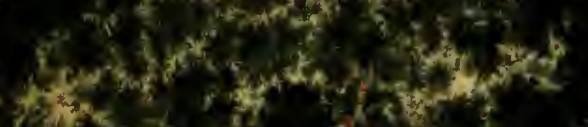

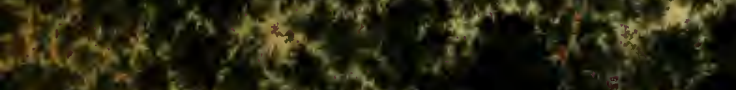
$x^{2}+4+3 \times$

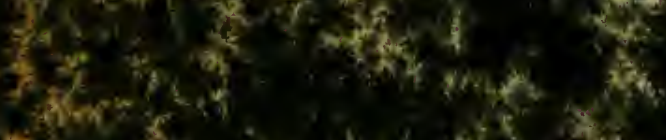

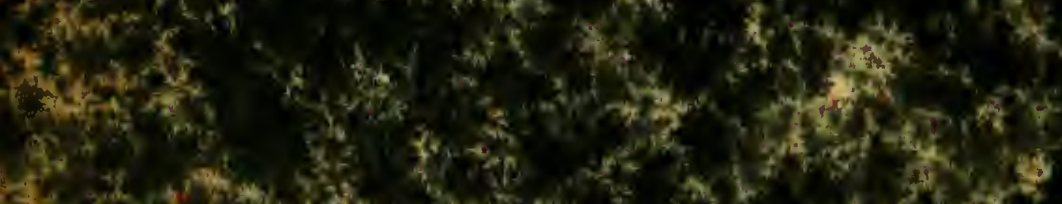

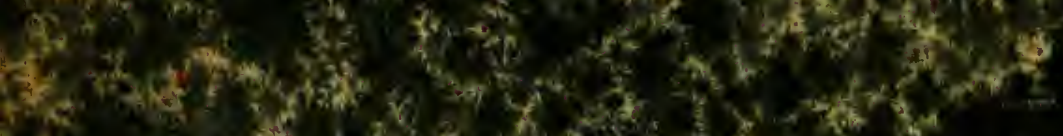

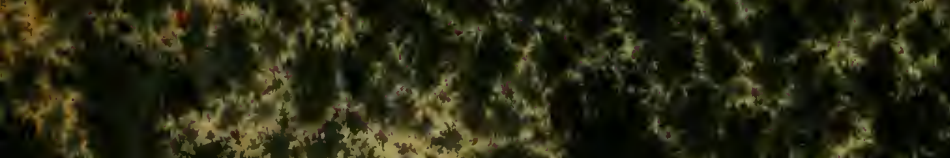
$x^{2}=x^{2}+x$, $\infty x$

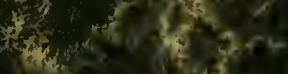

